\title{
Reidemeister torsion, peripheral complex and Alexander polynomials of hypersurface complements
}

\author{
YONGQIANG LIU \\ LAURENŢIU MAXIM
}

Let $f: \mathbb{C}^{n+1} \rightarrow \mathbb{C}$ be a polynomial that is transversal (or regular) at infinity. Let $\mathcal{U}=\mathbb{C}^{n+1} \backslash f^{-1}(0)$ be the corresponding affine hypersurface complement. By using the peripheral complex associated to $f$, we give several estimates for the (infinite cyclic) Alexander polynomials of $\mathcal{U}$ induced by $f$, and we describe the error terms for such estimates. The obtained polynomial identities can be further refined by using the Reidemeister torsion, generalizing a similar formula proved by Cogolludo and Florens in the case of plane curves. We also show that the above-mentioned peripheral complex underlies an algebraic mixed Hodge module. This fact allows us to construct mixed Hodge structures on the Alexander modules of the boundary manifold of $\mathcal{U}$.

$32 \mathrm{~S} 25 ; 32 \mathrm{~S} 55,32 \mathrm{~S} 60$

\section{Introduction}

\subsection{Background}

Let $f: \mathbb{C}^{n+1} \rightarrow \mathbb{C}$ be a polynomial map, and set $F_{0}=f^{-1}(0)$ and $\mathcal{U}=\mathbb{C}^{n+1} \backslash F_{0}$. The topological study of the hypersurface $F_{0}$ and of its complement $\mathcal{U}$ is a classical subject going back to Zariski. Libgober $[19 ; 20 ; 21]$ introduced and studied Alexandertype invariants associated to the hypersurface complement $\mathcal{U}$, as induced by $f$. For $F_{0}$ a plane curve $[19 ; 20]$, or a hypersurface with only isolated singularities, including at infinity [21], Libgober obtained a divisibility result, asserting that the only (possibly) non-trivial global Alexander polynomial of $\mathcal{U}$ divides the product of the local Alexander polynomials associated with each singular point (including at infinity).

More recently, the second author [25] used the intersection homology theory to provide generalizations of these results to the case of hypersurfaces with arbitrary singularities, provided that the defining equation $f$ is transversal at infinity (ie the hyperplane at infinity is generic with respect to the projective completion of $F_{0}$ ). In particular, he proved a general divisibility result (see [25, Theorem 4.2]) relating the global and local Alexander polynomials. Furthermore, Dimca and Libgober [10] showed that for 
a polynomial transversal at infinity there exist canonical mixed Hodge structures on the (torsion) Alexander invariants of the hypersurface complement. For more results related to Alexander-type invariants for complements of hypersurfaces with non-isolated singularities, see Dimca and Maxim [11] and Liu [23].

A different approach to the study of Alexander polynomials relies on the use of Reidemeister torsion. Milnor [26; 27] showed that the Alexander polynomial of a link coincides with the Reidemeister torsion of the link complement. This approach was further developed by Turaev [33] for the classical Alexander polynomial, and by Lin [22] and Wada [34] for twisted Alexander polynomials. Kirk and Livingston [18] extended this theory to any finite CW complex; in particular, they generalized Milnor's duality theorem for Reidemeister torsion.

Cogolludo and Florens [5] studied twisted Alexander polynomials of plane algebraic curves by using the Reidemeister torsion, and obtained a polynomial identity involving global and local twisted Alexander polynomials. Specializing their result to the classical case (of the trivial representation), one obtains a geometric interpretation of Libgober's divisibility result.

Let us assume from now on that $f$ is transversal at infinity. One of the goals of this paper is to provide a generalization to hypersurfaces with non-isolated singularities of the Cogolludo-Florens identity for Alexander polynomials (see [5, Corollary 5.8]). Our main tool will be the Cappell-Shaneson peripheral complex [4] associated to $f$. In more detail, we give a new description of the peripheral complex, from which we deduce several error estimates for the Alexander polynomials of the complement. Moreover, by exploiting the relation between the Alexander polynomials and Reidemeister torsion (see Kirk and Livingston [18, Theorem 3.4]), we show how these estimates can be further refined by using the intersection form appearing in the duality for Reidemeister torsion.

Our new description of the peripheral complex can also be used to show that the peripheral complex underlies an algebraic mixed Hodge module. In particular, after explaining the relation between the peripheral complex and the boundary manifold of the complement $\mathcal{U}$, we obtain mixed Hodge structures (MHS) on the Alexander modules of this boundary manifold.

\subsection{Main results}

Unless otherwise specified, all homology and cohomology groups will be assumed to have $\mathbb{Q}$-coefficients.

Let $f: \mathbb{C}^{n+1} \rightarrow \mathbb{C}$ be a degree- $d$ polynomial. We say that $f$ is transversal (or regular) at infinity if $f$ is reduced and the projective closure $V$ of $F_{0}$ in $\mathbb{C P}^{n+1}$ is transversal 
in the stratified sense to the hyperplane at infinity $H=\mathbb{C P}^{n+1} \backslash \mathbb{C}^{n+1}$. Consider the infinite cyclic cover $\mathcal{U}^{c}$ of $\mathcal{U}$ corresponding to the kernel of the linking number homomorphism

$$
f_{*}: \pi_{1}(\mathcal{U}) \rightarrow \pi_{1}\left(\mathbb{C}^{*}\right)=\mathbb{Z}
$$

induced by $f$. Then, under the deck group action, each homology group $H_{i}\left(\mathcal{U}^{c}\right)$ becomes a $\Gamma:=\mathbb{Q}\left[t, t^{-1}\right]$-module, called the $i^{\text {th }}$ Alexander module of the hypersurface complement $\mathcal{U}$. For $f$ transversal at infinity, Maxim [25, Theorem 3.6] showed that $H_{i}\left(\mathcal{U}^{c}\right)$ is a torsion $\Gamma$-module for $i \leq n$. We denote by $\delta_{i}(t)$ the corresponding (global) Alexander polynomial.

Let $N$ be an open regular neighbourhood of $V \cup H$ in $\mathbb{C P}^{n+1}$ (see Durfee [14]). Set $\mathcal{U}_{0}=\mathbb{C P}^{n+1} \backslash N$. Then $\mathcal{U}_{0}$ is homotopy equivalent to $\mathcal{U}$, and the boundary $\partial \mathcal{U}_{0}$ is a $(2 n+1)$-dimensional real manifold, called the boundary manifold of $\mathcal{U}$. The inclusion $\partial \mathcal{U}_{0} \hookrightarrow \mathcal{U}_{0}$ is an $n$-homotopy equivalence (see Dimca [8, Proposition (5.2.31)]). Moreover, we have an epimorphism

$$
\rho: \pi_{1}\left(\partial \mathcal{U}_{0}\right) \longrightarrow \pi_{1}\left(\mathcal{U}_{0}\right)=\pi_{1}(\mathcal{U}) \stackrel{f_{*}}{\longrightarrow} \pi_{1}\left(\mathbb{C}^{*}\right)=\mathbb{Z},
$$

which defines the infinite cyclic cover $\left(\partial \mathcal{U}_{0}\right)^{c}$ of $\partial \mathcal{U}_{0}$. The related intersection form $\phi^{\rho} \in \mathbb{Q}(t)$ for the pair $\left(\mathcal{U}_{0}, \partial \mathcal{U}_{0}\right)$ is defined on $H_{n+1}\left(\mathcal{U}_{0}^{c}\right)$; see [18] or Section 5.3 below.

The peripheral complex $\mathcal{R}^{\bullet}$ associated to $f$ (see [4; 25] or Definition 2.5 below) is a torsion $\Gamma$-module sheaf complex, which plays a key role in Maxim's generalizations of Libgober's results to the case of hypersurfaces with non-isolated singularities. Our first result is the following (see Proposition 6.1 and Corollaries 3.3 and 6.2):

Theorem 1.1 Assume that the polynomial $f: \mathbb{C}^{n+1} \rightarrow \mathbb{C}$ is transversal at infinity. Then:

(a) There are $\Gamma$-module isomorphisms

$$
H_{i}\left(\left(\partial \mathcal{U}_{0}\right)^{c}\right) \cong H^{2 n+1-i}\left(\mathbb{C P}^{n+1} ; \mathcal{R}^{\bullet}\right) \text { for all } i
$$

and, in particular, $H_{i}\left(\left(\partial \mathcal{U}_{0}\right)^{c}\right)$ is a torsion $\Gamma$-module. Moreover, the zeros of the Alexander polynomial associated to $H_{i}\left(\left(\partial \mathcal{U}_{0}\right)^{c}\right)$ are roots of unity for all $i$, and have order $d$ except for $i=n$. Finally, $H_{i}\left(\left(\partial \mathcal{U}_{0}\right)^{c}\right)$ is a semi-simple $\Gamma$-module for $i \neq n$.

(b) The peripheral complex $\mathcal{R} \bullet$ (when regarded as a complex of $\mathbb{Q}$-vector sheaves) is a (shifted) mixed Hodge module, hence the vector spaces $H_{i}\left(\left(\partial \mathcal{U}_{0}\right)^{c}\right)$ inherit mixed Hodge structures from $\mathcal{R}^{\bullet}$ for all $i$. Moreover, for $i \neq n$, the mixed Hodge structure on $H_{i}\left(\left(\partial \mathcal{U}_{0}\right)^{c}\right)$ is compatible with the $\Gamma$-action, ie $t: H_{i}\left(\left(\partial \mathcal{U}_{0}\right)^{c}\right) \rightarrow H_{i}\left(\left(\partial \mathcal{U}_{0}\right)^{c}\right)$ is a mixed Hodge structure homomorphism. 
Let $h=f_{d}$ be the top-degree part of $f$, with corresponding Milnor fibre $F_{h}=\{h=1\}$, and denote by $h_{i}(t)$ the Alexander polynomial (or order) associated to the torsion $\Gamma$-module $H_{i}\left(F_{h}\right)$. On the other hand, let $\psi_{f} \mathbb{Q}_{\mathbb{C}^{n+1}}$ be the nearby cycle complex associated to $f$, and denote by $\psi_{i}(t)$ the corresponding Alexander polynomial of $H_{c}^{2 n+1-i}\left(F_{0}, \psi_{f} \mathbb{Q}_{\mathbb{C}^{n+1}}\right)$. Liu [23, Theorem 1.1] studied the relation between the polynomials $\psi_{i}(t)$ and the Alexander polynomials $\delta_{i}(t)$ of the hypersurface complement $\mathcal{U}$. In particular, he showed that $\psi_{i}(t)=\delta_{i}(t)$ for $i<n$, and $\delta_{n}(t)$ divides $\psi_{n}(t)$. With the above notations, we have the following result, which establishes a more precise relationship between the polynomials $\delta_{n}(t)$ and $\psi_{n}(t)$ (see Theorem 7.1):

Theorem 1.2 Assume that the degree-d polynomial $f: \mathbb{C}^{n+1} \rightarrow \mathbb{C}$ is transversal at infinity. Let $\phi^{\rho}$ be the intersection form for $\left(\mathcal{U}_{0}, \partial \mathcal{U}_{0}\right)$ induced by $\rho$. Then

$$
h_{n}(t) \cdot \psi_{n}(t)=\delta_{n}^{2}(t) \cdot \operatorname{det}\left(\phi^{\rho}\right)
$$

Moreover, we have the degree estimates ${ }^{1}$

$$
\operatorname{deg}\left(\operatorname{det}\left(\phi^{\rho}\right)\right) \leq 2 d \cdot \mu
$$

where $\mu=|\chi(\mathcal{U})|$ is the absolute value of the Euler characteristic of $\mathcal{U}$.

As an application to the case of polynomials with only isolated singularities, we obtain the following generalization of [5, Corollary 5.8], and a new obstruction on the (degree of the) intersection form:

Corollary 1.3 Let $f: \mathbb{C}^{n+1} \rightarrow \mathbb{C}$ be a degree-d polynomial that is transversal at infinity. Assume that the hypersurface $F_{0}=\{f=0\}$ has only isolated singularities. Then we have the polynomial identity

$$
(t-1)^{(-1)^{n+1}(1+\chi(\mathcal{U}))}\left(t^{d}-1\right)^{\xi} \cdot \prod_{p \in \operatorname{Sing}\left(F_{0}\right)} \Delta_{p}(t)=\delta_{n}(t)^{2} \cdot \operatorname{det}\left(\phi^{\rho}\right),
$$

where $\Delta_{p}(t)$ is the top local Alexander polynomial associated to the point $p \in \operatorname{Sing}\left(F_{0}\right)$ and $\xi=\left((d-1)^{n+1}+(-1)^{n}\right) / d$. Moreover, the degree of the polynomial $\operatorname{det}\left(\phi^{\rho}\right)$ is even.

\footnotetext{
${ }^{1}$ Recall that the total degree of a Laurent polynomial in $\mathbb{Q}\left[t, t^{-1}\right]$ is defined as the difference between the highest and the lowest power of $t$ (with non-zero coefficients). In particular, unit elements $c t^{k}(c \in \mathbb{Q}$, $k \in \mathbb{Z}$ ) of $\mathbb{Q}\left[t, t^{-1}\right]$ have total degree zero. The total degree of a product of Laurent polynomials is the sum of the total degrees of the factors. The degree of an element $P / Q \in \mathbb{Q}(t)$ (with $P, Q \in \mathbb{Q}\left[t, t^{-1}\right]$ ) is defined as the difference between the total degrees of $P$ and $Q$.
} 


\subsection{Summary}

The paper is organized as follows.

In Section 2, we recall the definition and main results on the Alexander modules, peripheral complex and the Sabbah specialization complex. In Section 3, we give a new description of the peripheral complex associated with a hypersurface. As a byproduct, we show that the peripheral complex underlies a (shifted) algebraic mixed Hodge module. In Section 4, we give several estimates for the Alexander polynomials of the hypersurface complement and study the error terms for such estimates. Section 5 recalls the basic constructions and main results on the Reidemeister torsion of a finite CW complex, and in particular, the duality theorem and the intersection form for the torsion. In Section 6, we introduce the boundary manifold $\partial \mathcal{U}_{0}$ of the hypersurface complement, and we describe its (linking number) Alexander modules $H_{i}\left(\left(\partial \mathcal{U}_{0}\right)^{c}\right)$ in terms of the peripheral complex. In particular, we endow these Alexander modules $H_{i}\left(\left(\partial \mathcal{U}_{0}\right)^{c}\right)$ with mixed Hodge structures. Finally, Section 7 is devoted to the proof of both Theorem 1.2 and Corollary 1.3.

\section{Acknowledgments}

We are grateful to Alex Dimca and Jörg Schürmann for useful discussions. Liu is supported by China Scholarship Council (file No 201206340046). He thanks the Mathematics Department at the University of Wisconsin-Madison for hospitality during the preparation of this work. Maxim is partially supported by grants from NSF (DMS1304999), NSA (H98230-14-1-0130), Simons Foundation (\#277891), and by a grant of the Ministry of National Education, CNCS-UEFISCDI project number PN-II-IDPCE-2012-4-0156.

\section{Preliminaries}

\subsection{Alexander modules}

Let $f=f\left(x_{1}, \ldots, x_{n+1}\right): \mathbb{C}^{n+1} \rightarrow \mathbb{C}$ be a reduced degree- $d$ polynomial map, and set $F_{0}=f^{-1}(0)$ and $\mathcal{U}=\mathbb{C}^{n+1} \backslash F_{0}$. We say that $f$ is transversal at infinity if the projective closure $V$ of $F_{0}$ in $\mathbb{C P}^{n+1}$ is transversal in the stratified sense to the hyperplane at infinity $H=\mathbb{C P}^{n+1} \backslash \mathbb{C}^{n+1}=\left\{x_{0}=0\right\}$. If $f$ is transversal at infinity, the affine hypersurface $F_{0}$ is homotopy equivalent to a bouquet of $n$-spheres, ie

$$
F_{0} \simeq \bigvee_{\mu} S^{n}
$$


where $\mu$ denotes the number of spheres in the join (see [13, page 476]). It is shown there that $\mu$ can be determined topologically as the degree of the gradient map associated to the homogenization $\tilde{f}$ of $f$.

We have a surjective homomorphism: $\pi_{1}(\mathcal{U}) \rightarrow \pi_{1}\left(\mathbb{C}^{*}\right)=\mathbb{Z}$ induced by $f$, which shall be called the linking number homomorphism (see [8, pages 76-77] for a justification of terminology). Let us consider the corresponding infinite cyclic cover $\mathcal{U}^{c}$ of $\mathcal{U}$. Then, under the deck group action, every homology group $H_{i}\left(\mathcal{U}^{c}, \mathbb{Q}\right)$ becomes a $\Gamma:=\mathbb{Q}\left[t, t^{-1}\right]$-module.

Definition 2.1 The $\Gamma$-module $H_{i}\left(\mathcal{U}^{c}\right)$ is called the $i^{\text {th }}$ Alexander module of the hypersurface complement $\mathcal{U}$.

When $H_{i}\left(\mathcal{U}^{c}\right)$ is a torsion $\Gamma$-module, we denote by $\delta_{i}(t)$ the corresponding Alexander polynomial (also called order by Milnor [28]). Since $\mathcal{U}$ has the homotopy type of a finite $(n+1)$-dimensional CW complex, $H_{i}\left(\mathcal{U}^{c}\right)=0$ for $i>n+1$ and $H_{n+1}\left(\mathcal{U}^{c}\right)$ is a free $\Gamma$-module. Hence the only interesting Alexander modules $H_{i}\left(\mathcal{U}^{c}\right)$ appear in the range $0 \leq i \leq n$, and the following result holds:

Theorem 2.2 [25, Theorems 3.6 and 4.1] Assume that $f: \mathbb{C}^{n+1} \rightarrow \mathbb{C}$ is a reduced, degree- $d$ polynomial that is transversal at infinity. Then $H_{i}\left(\mathcal{U}^{c}\right)$ is a finitely generated semi-simple torsion $\Gamma$-module for $0 \leq i \leq n$, and the roots of the corresponding Alexander polynomial $\delta_{i}(t)$ are roots of unity of order $d$.

Remark 2.3 Maxim [25] showed that $H_{0}\left(\mathcal{U}^{c}\right) \cong \Gamma /(t-1)$, and $H_{n+1}\left(\mathcal{U}^{c}\right)$ is a free $\Gamma$-module of rank $|\chi(\mathcal{U})|$. On the other hand, by using the additivity of the Euler characteristic, it is easy to see from $(2-1)$ that $\chi(\mathcal{U})=(-1)^{n+1} \mu$. Therefore

$$
H_{n+1}\left(\mathcal{U}^{c}\right) \cong \Gamma^{\mu} \text {. }
$$

\subsection{Linking number local system}

Let us consider the local system $\mathcal{L}$ on $\mathcal{U}$ with stalk $\Gamma$, and representation of the fundamental group defined by the composition

$$
\pi_{1}(\mathcal{U}) \stackrel{f_{*}}{\longrightarrow} \pi_{1}\left(\mathbb{C}^{*}\right) \longrightarrow \operatorname{Aut}(\Gamma),
$$

with the second map being given by $1_{\mathbb{Z}} \mapsto t$. Here $t$ is the automorphism of $\Gamma$ given by multiplication by $t . \mathcal{L}$ shall be referred to as the linking number local system.

If $A^{\bullet}$ is a complex of $\Gamma$-sheaves, let $\mathcal{D} A^{\bullet}$ denote its Verdier dual. Then we have that

$$
\mathcal{D} \mathcal{L} \cong \mathcal{L}^{\mathrm{op}}[2 n+2]
$$


where $\mathcal{L}^{\text {op }}$ is the local system obtained from $\mathcal{L}$ by composing all $\Gamma$-module structures with the involution $t \mapsto t^{-1}$.

In terms of the local system $\mathcal{L}$, we have the $\Gamma$-module isomorphisms [25, Corollary 3.4]

$$
H_{c}^{2 n+2-i}(\mathcal{U}, \mathcal{L}) \cong H_{i}(\mathcal{U}, \mathcal{L}) \cong H_{i}\left(\mathcal{U}^{c}\right)
$$

for all $i$. Similarly,

$$
H_{i}\left(\mathcal{U}, \mathcal{L}^{\mathrm{op}}\right) \cong \overline{H_{i}\left(\mathcal{U}^{c}\right)}
$$

where $\bar{*}$ denotes the composition with the involution $t \mapsto t^{-1}$. By using the universal coefficient theorem (eg see Banagl [1, Theorem 3.4.4]), we also obtain

$$
H^{i+1}(\mathcal{U} ; \mathcal{L}) \cong \operatorname{Free}\left(H_{i+1}\left(\mathcal{U}, \mathcal{L}^{\mathrm{op}}\right)\right) \oplus \operatorname{Torsion}\left(H_{i}\left(\mathcal{U}, \mathcal{L}^{\mathrm{op}}\right)\right)
$$

\subsection{The peripheral complex}

For any complex algebraic variety $X$ and any ring $R$, we denote by $D_{c}^{b}(X, R)$ the derived category of bounded, cohomologically $R$-constructible complexes of sheaves on $X$. For a quick introduction to derived categories, the reader is advised to consult Dimca [9].

By choosing a Whitney stratification of $V$ and using the transversal hyperplane at infinity $H$, we obtain a stratification of the pair $\left(\mathbb{C P}^{n+1}, V \cup H\right)$. Then, for any perversity function $\bar{p}$, the intersection homology complex $I C_{\bar{p}}^{\bullet}\left(\mathbb{C P}^{n+1}, \mathcal{L}\right) \in D_{c}^{b}\left(\mathbb{C P}^{n+1}, \Gamma\right)$ is defined by using Deligne's axiomatic construction (see Banagl [1] or Goresky and MacPherson [15]). In this paper, we mainly use the indexing conventions from [15]. In particular, we have the normalization property $\left.I C_{\bar{p}}^{\bullet}\left(\mathbb{C P}^{n+1}, \mathcal{L}\right)\right|_{\mathcal{U}} \cong \mathcal{L}[2 n+2]$.

Let us recall the following result:

Theorem 2.4 [25, Lemma 3.1] Assume that the polynomial $f: \mathbb{C}^{n+1} \rightarrow \mathbb{C}$ is transversal at infinity. Let $j$ be the inclusion of $\mathcal{U}$ in $\mathbb{C P}^{n+1}$. Then we have quasiisomorphisms in $D_{c}^{b}\left(\mathbb{C P}^{n+1}, \Gamma\right)$

$$
\begin{aligned}
I C_{\bar{m}}^{\bullet}\left(\mathbb{C P}^{n+1}, \mathcal{L}\right) & \cong j_{!} \mathcal{L}[2 n+2], \\
I C_{\bar{l}}^{\bullet}\left(\mathbb{C P}^{n+1}, \mathcal{L}\right) & \cong R j_{*} \mathcal{L}[2 n+2],
\end{aligned}
$$

where the middle and logarithmic perversities are defined as $\bar{m}(s)=[(s-1) / 2]$ and $\bar{l}(s)=[(s+1) / 2]$. (Note that $\bar{m}(s)+\bar{l}(s)=s-1$, ie $\bar{m}$ and $\bar{l}$ are superdual perversities, in the sense of [4].) 
In the above notations, the Cappell-Shaneson superduality isomorphism can be stated as (see [4, Theorem 3.3])

$$
I C_{\bar{m}}^{\bullet}\left(\mathbb{C P}^{n+1}, \mathcal{L}\right)^{\mathrm{op}} \cong \mathcal{D}\left(I C_{\bar{l}}^{\bullet}\left(\mathbb{C P}^{n+1}, \mathcal{L}\right)\right)[2 n+2],
$$

where, if $A$ is a complex of sheaves, $A^{\text {op }}$ is the $\Gamma$-module obtained from the $\Gamma$-module $A$ by composing all module structures with the involution $t \mapsto t^{-1}$.

Definition 2.5 The peripheral complex $\mathcal{R}^{\bullet} \in D_{c}^{b}\left(\mathbb{C P}^{n+1}, \Gamma\right)$ is defined by the distinguished triangle (see [4])

$$
I C_{\bar{m}}^{\bullet}\left(\mathbb{C P}^{n+1}, \mathcal{L}\right) \rightarrow I C_{\bar{l}}^{\bullet}\left(\mathbb{C P}^{n+1}, \mathcal{L}\right) \rightarrow \mathcal{R} \cdot[2 n+2] \stackrel{[1]}{\rightarrow},
$$

or, using Theorem 2.4, by

$$
j ! \mathcal{L} \rightarrow R j_{*} \mathcal{L} \rightarrow \mathcal{R}^{\bullet} \stackrel{[1]}{\rightarrow}
$$

Then, up to a shift, $\mathcal{R}^{\bullet}$ is a self-dual (ie $\mathcal{R}^{\bullet} \cong \mathcal{D} \mathcal{R}^{\bullet \text { op }}[-2 n-1]$ ), torsion (ie the stalks of its cohomology sheaves are torsion modules), perverse sheaf on $\mathbb{C P}^{n+1}$ (see [25, Section 3.2]). In fact, $\mathcal{R}^{\bullet}$ has compact support on $V \cup H$ and

$$
\left.\left.\mathcal{R}^{\bullet}\right|_{V \cup H} \cong\left(R j_{*} \mathcal{L}\right)\right|_{V \cup H} .
$$

Remark 2.6 The peripheral complex $\mathcal{R}^{\bullet}$ as defined here corresponds to $\mathcal{R}^{\bullet}[-2 n-2]$ in the notations of Cappell and Shaneson; see [4] or [25].

\subsection{The Sabbah specialization complex}

The Sabbah specialization complex [29] (see also its reformulation by Budur [3]) can be regarded as a generalization of Deligne's nearby cycle complex. For a quick introduction to the theory of nearby cycles, the reader is advised to consult Dimca [9] and Massey [24].

Let us recall the relevant definitions. Consider the commutative diagram of spaces and maps

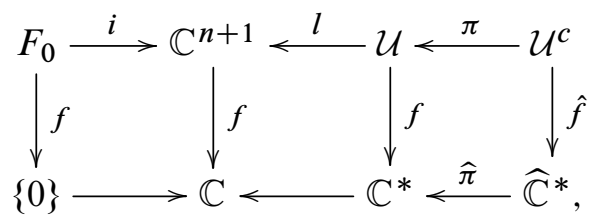

where $\hat{\pi}$ is the universal covering of the punctured disk $\mathbb{C}^{*}$ and the right-hand square of the diagram is cartesian. 
Definition 2.7 The Sabbah specialization functor of $f$ is defined by

$$
\psi_{f}^{S}=i^{*} R l_{*} R \pi_{!}(l \circ \pi)^{*}: D_{c}^{b}\left(\mathbb{C}^{n+1}, \mathbb{Q}\right) \rightarrow D_{c}^{b}\left(F_{0}, \Gamma\right),
$$

and we call $\psi_{f}^{S} \mathbb{Q}_{\mathbb{C}^{n+1}}$ the Sabbah specialization complex.

Remark 2.8 The definition of the Sabbah specialization complex is slightly different from that of the nearby cycle complex, where $R \pi_{\text {! }}$ is replaced by $R \pi_{*}$.

In the following we write $\mathbb{Q}$ for the constant sheaf $\mathbb{Q}_{\mathbb{C}^{n+1}}$ on $\mathbb{C}^{n+1}$.

Consider the natural forgetful functor

$$
\text { for: } D_{c}^{b}\left(F_{0}, \Gamma\right) \rightarrow D_{c}^{b}\left(F_{0}, \mathbb{Q}\right),
$$

which maps a torsion $\Gamma$-module sheaf complex to its underlying $\mathbb{Q}$-complex. Let $\psi_{f} \mathbb{Q}$ be the Deligne nearby cycle complex associated to $f$. It is known that one has a non-canonical isomorphism (see [2, page 13])

$$
\text { for } \circ \psi_{f}^{S}(\mathbb{Q}) \cong \psi_{f} \mathbb{Q}[-1] .
$$

The next result is a direct consequence of [3, Lemma 3.4(b)].

Lemma 2.9 [23, Section 2.4] We have a quasi-isomorphism in $D_{c}^{b}\left(F_{0}, \Gamma\right)$,

$$
\left.\mathcal{R}^{\bullet}\right|_{F_{0}} \cong \psi_{f}^{S} \mathbb{Q}
$$

Moreover, we have the distinguished triangle in $D_{c}^{b}\left(\mathbb{C}^{n+1}, \Gamma\right)$,

$$
l_{!} \mathcal{L} \rightarrow R l_{*} \mathcal{L} \rightarrow i_{!} \psi_{f}^{S} \stackrel{[1]}{\rightarrow}
$$

\section{Peripheral complex as a mixed Hodge module}

In this section, we give a new characterization of the peripheral complex and show that (up to a shift) it underlies a mixed Hodge module. For a quick introduction to the category of mixed Hodge module, the reader is advised to consult Saito [30].

Let $h=f_{d}$ be the top-degree part of $f$, with corresponding Milnor fibre $F_{h}=\{h=1\}$. Then, it is shown by Maxim [25] that $\left.\mathcal{R} \bullet\right|_{H \backslash H \cap V}$ is a local system $\mathcal{L}(h)$ with stalk $\Gamma /\left(t^{d}-1\right)$ placed in degree 1 , ie

$$
\left.\mathcal{R}^{\bullet}\right|_{H \backslash H \cap V} \cong \mathcal{L}(h)[-1] .
$$


Theorem 3.1 Assume that the polynomial $f: \mathbb{C}^{n+1} \rightarrow \mathbb{C}$ is transversal at infinity and let $V$ be the projective completion of $F_{0}=\{f=0\}$. Let $i_{v}$ be the inclusion of $F_{0}$ into $V$ and $i_{h}$ be the inclusion of $H \backslash V \cap H$ into $H$. Then

$$
\begin{aligned}
\left.\mathcal{R}^{\bullet}\right|_{V} \cong R i_{v *} \psi_{f}^{S} \mathbb{Q}, \\
\left.\mathcal{R}^{\bullet}\right|_{H} \cong R i_{h *} \mathcal{L}(h)[-1] .
\end{aligned}
$$

Proof Let us only prove (3-2), as (3-3) can be obtained in a similar manner. Consider the following commutative diagram of inclusions:

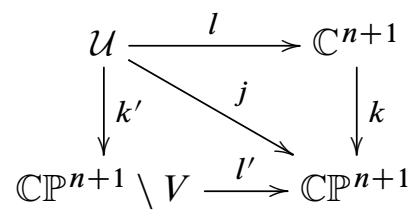

Since $V$ intersects $H$ transversally, there exists a base change isomorphism associated with this diagram (see Schürmann [32, Lemma 6.0.5]),

$$
l_{!}^{\prime} \circ R k_{*}^{\prime}=R k_{*} \circ l_{!} .
$$

Let $v$ be the inclusion of $V$ into $\mathbb{C P}^{n+1}$. Note that $R l_{*}^{\prime} R k_{*}^{\prime} \mathcal{L}=R j_{*} \mathcal{L}$ and, by Section 2.3, we have that $\left.\mathcal{R} \bullet\right|_{V}=\left.\left(R j_{*} \mathcal{L}\right)\right|_{V}$. Then we have a distinguished triangle

$$
l_{!}^{\prime} R k_{*}^{\prime} \mathcal{L} \rightarrow R l_{*}^{\prime} k_{*}^{\prime} \mathcal{L} \rightarrow v_{!}\left(\left.\mathcal{R}\right|_{V}\right) \stackrel{[1]}{\rightarrow}
$$

Using the base change isomorphism (3-4) and the commutativity of the above diagram, the distinguished triangle (3-5) can be written as

$$
R k_{*} l_{!} \mathcal{L} \rightarrow R k_{*} R l_{*} \mathcal{L} \rightarrow v_{!}\left(\left.\mathcal{R}^{\bullet}\right|_{V}\right) \stackrel{[1]}{\rightarrow}
$$

Recall now that there is a distinguished triangle (2-14)

$$
l_{!} \mathcal{L} \rightarrow R l_{*} \mathcal{L} \rightarrow i_{!} \psi_{f}^{S} \mathbb{Q}
$$

where $i$ is the inclusion of $F_{0}$ into $\mathbb{C}^{n+1}$. By applying the functor $R k_{*}$ to this triangle, we obtain the distinguished triangle

$$
R k_{*} l_{!} \mathcal{L} \rightarrow R k_{*} R l_{*} \mathcal{L} \rightarrow R k_{*} i_{!} \psi_{f}^{S} \mathbb{Q} \stackrel{[1]}{\rightarrow} .
$$

So, by comparing the two triangles (3-6) and (3-8), we get the quasi-isomorphism

$$
v_{!}\left(\left.\mathcal{R}^{\bullet}\right|_{V}\right) \cong R k_{*} i_{!} \psi_{f}^{S} \mathbb{Q}
$$


Since $F_{0}$ is closed in $\mathbb{C}^{n+1}$, we have $i_{!}=R i_{*}$. So, for $i_{v}$ the inclusion of $F_{0}$ into $V$, we have $v \circ i_{v}=k \circ i$, hence

$$
R k_{*} i_{!} \psi_{f}^{S} \mathbb{Q}=R k_{*} R i_{*} \psi_{f}^{S} \mathbb{Q}=R v_{*} R i_{v *} \psi_{f}^{S} \mathbb{Q} .
$$

Finally, by applying $v^{*}$ to (3-9), and using (3-10) and the standard identities $v^{*} v_{!}=$id and $v^{*} R v_{*}=\mathrm{id}$, we get

$$
\left.\mathcal{R}^{\bullet}\right|_{V} \cong R i_{v *} \psi_{f}^{S} \mathbb{Q}
$$

The result of Theorem 3.1 above can be used to endow the peripheral complex with a mixed Hodge module structure. Recall that there is a natural forgetful functor for: $D_{c}^{b}(X, \Gamma) \rightarrow D_{c}^{b}(X, \mathbb{Q})$, which assigns to a torsion complex of $\Gamma$-sheaves its underlying $\mathbb{Q}$-complex. In what follows, we will use the same notation for a $\Gamma$-complex $\mathcal{A}^{\bullet}$ and for its underlying $\mathbb{Q}$-complex $\operatorname{for}\left(\mathcal{A}^{\bullet}\right)$.

After applying the forgetful functor to (3-2) and using (2-12), we obtain the quasiisomorphism of complexes of $\mathbb{Q}$-sheaves

$$
\left.\mathcal{R}^{\bullet}\right|_{V} \cong R i_{v *} \psi_{f} \mathbb{Q}[-1] .
$$

Also note that the local system $\mathcal{L}(h) \in D_{c}^{b}(H \backslash H \cap V, \mathbb{Q})$ is induced by the natural $d$-fold cover map $p: F_{h} \rightarrow(H \backslash V \cap H)$ or, more precisely,

$$
\mathcal{L}(h) \cong R p_{*} \mathbb{Q}_{F_{h}} \in D_{c}^{b}(H \backslash H \cap V, \mathbb{Q}) .
$$

Remark 3.2 Since $h=f_{d}$ is a degree- $d$ homogeneous polynomial function on $\mathbb{C}^{n+1}$, the hypersurface $V_{h}=\overline{\{h=0\}} \subseteq \mathbb{C P}^{n+1}$ is already transversal to the hyperplane at infinity $H=\left\{x_{0}=0\right\}$. Let $\mathcal{R}_{h}^{\bullet}$ be the peripheral complex associated to $h$. Then (3-3) implies that $\left.\mathcal{R}^{\bullet}\right|_{H}=\left.\mathcal{R}_{h}^{\bullet}\right|_{H}$.

We can now prove the following result:

Corollary 3.3 The peripheral complex $\mathcal{R}^{\bullet}$ underlies a (shifted) algebraic mixed Hodge module.

Proof For the purpose of this proof only, we switch to the perverse conventions used in Saito's theory, according to which $\mathcal{R}^{\bullet}[n+1]$ is a perverse sheaf on $\mathbb{C P}^{n+1}$. All sheaf complexes appearing in this proof will be complexes of $\mathbb{Q}$-sheaves (ie we apply the forgetful functor to all $\Gamma$-sheaf complexes).

Consider the inclusions $H \backslash V \cap H \stackrel{s}{\hookrightarrow} V \cup H \stackrel{r}{\leftrightarrow} V$, and the associated distinguished triangle in $D_{c}^{b}(V \cup H, \mathbb{Q})$

$$
\left.\left.s ! \mathcal{R}^{\bullet}\right|_{H \backslash V \cap H}[n+1] \rightarrow \mathcal{R}^{\bullet}[n+1] \rightarrow r_{*} \mathcal{R}^{\bullet}\right|_{V}[n+1] \stackrel{[1]}{\rightarrow} .
$$


Recall that $\left.\mathcal{R}^{\bullet}\right|_{H \backslash V \cap H} \cong \mathcal{L}(h)[-1]$, while by (3-12) we have $\left.\mathcal{R}^{\bullet}\right|_{V} \cong R i_{v *} \psi_{f} \mathbb{Q}[-1]$. Since $\left.\mathcal{R}^{\bullet}\right|_{H \backslash V \cap H}[n+1] \cong \mathcal{L}(h)[n]$ is a perverse sheaf on $H \backslash V \cap H$ and $s$ is a quasi-finite affine map, it follows from [9, Corollary 5.2.17] that $\left.s ! \mathcal{R} \bullet\right|_{H \backslash V \cap H}[n+1]$ is a perverse sheaf on $V \cup H$. Moreover, we deduce by (3-13) that $\mathcal{L}(h)[n]$, hence also $\left.s ! \mathcal{R}^{\bullet}\right|_{H \backslash V \cap H}[n+1]$, underlie algebraic mixed Hodge modules. Since $\mathbb{Q}[n+1]$ is a perverse sheaf on $\mathbb{C}^{n+1}$ underlying a mixed Hodge module, and the functor $\psi_{f}[-1]$ preserves perverse sheaves (and mixed Hodge modules), it follows that $\left(\psi_{f}[-1]\right)(\mathbb{Q}[n+1])$ is a perverse sheaf on $F_{0}$ underlying a mixed Hodge module. Moreover, as $i_{v}$ is a quasifinite affine morphism, it follows as above that $\left.\mathcal{R} \bullet\right|_{V}[n+1] \cong R i_{v *}\left(\psi_{f}[-1]\right)(\mathbb{Q}[n+1])$ is a perverse sheaf on $V$ underlying a mixed Hodge module. Finally, since $r$ is proper, $r_{!}=r_{*}$ preserves perverse sheaves and mixed Hodge modules, so $\left.r_{*} \mathcal{R} \bullet\right|_{V}[n+1]$ is a perverse sheaf on $V \cup H$ underlying a mixed Hodge module.

The above considerations show that $\mathcal{R}^{\bullet}[n+1]$ can be regarded as an extension of perverse sheaves, both of which underlie mixed Hodge modules. So $\mathcal{R} \bullet[n+1]$ is an element in the first Yoneda extension group $Y \operatorname{Ext}^{1}(\operatorname{For}(C), \operatorname{For}(A))$ for suitable mixed Hodge modules $C$ and $A$ as described above, where For: MHM $\rightarrow$ Perv $_{\mathbb{Q}}$ denotes the forgetful functor assigning to a mixed Hodge module the corresponding rational sheaf complex. Since Yoneda Ext groups $Y \mathrm{Ext}^{i}$ agree with the derived category Ext groups $\operatorname{Ext}^{i}=\operatorname{Hom}(-,-[i])$ for noetherian or artinian abelian categories such as MHM or $\operatorname{Perv}_{\mathbb{Q}}$, and the forgetful functor

$$
\text { For: } \operatorname{Ext}^{i}(C, A) \rightarrow \operatorname{Ext}^{i}(\operatorname{For}(C), \operatorname{For}(A))
$$

is surjective for all $i$ for given mixed Hodge modules $A$ and $C$ (see [31, Theorem 2.10]), it follows that $\mathcal{R}^{\bullet}[n+1]$ underlies a mixed Hodge module.

\section{Error estimates for Alexander polynomials}

In this section, we give several error estimates for Alexander polynomials of hypersurface complements.

Proposition 4.1 In our notations, we have $\Gamma$-module isomorphisms

$$
H^{2 n+1-i}\left(\mathbb{C P}^{n+1} ; \mathcal{R}^{\bullet}\right) \cong \begin{cases}\frac{H_{i}\left(\mathcal{U}^{c}\right)}{H_{2 n-i}\left(\mathcal{U}^{c}\right)} & i>n,\end{cases}
$$

and an exact sequence of $\Gamma$-modules for $i=n$ :

$$
0 \rightarrow \Gamma^{\mu} \rightarrow \Gamma^{\mu} \oplus \overline{H_{n}\left(\mathcal{U}^{c}\right)} \rightarrow H^{n+1}\left(\mathbb{C P}^{n+1} ; \mathcal{R}^{\bullet}\right) \rightarrow H_{n}\left(\mathcal{U}^{c}\right) \rightarrow 0
$$


Proof Consider the distinguished triangle

$$
R j_{!} \mathcal{L} \rightarrow R j_{*} \mathcal{L} \rightarrow \mathcal{R} \bullet \stackrel{[1]}{\rightarrow}
$$

of Definition 2.5. By applying the hypercohomology with compact support functor, we have the long exact sequence

$$
\begin{aligned}
\cdots \rightarrow H^{2 n+1-i}\left(\mathbb{C P}^{n+1} ; \mathcal{R}^{\bullet}\right) & \rightarrow H^{2 n+2-i}\left(\mathbb{C P}^{n+1} ; R j ! \mathcal{L}\right) \\
& \rightarrow H^{2 n+2-i}\left(\mathbb{C P}^{n+1} ; R j_{*} \mathcal{L}\right) \rightarrow \cdots
\end{aligned}
$$

The claim follows from the above sequence together with the following $\Gamma$-isomorphisms from Section 2.2:

$$
\begin{aligned}
H^{2 n+2-i}\left(\mathbb{C P}^{n+1} ; R j_{!} \mathcal{L}\right) & \cong H_{c}^{2 n+2-i}(\mathcal{U} ; \mathcal{L}) \cong H_{i}\left(\mathcal{U}^{c}\right) \\
H^{2 n+2-i}\left(\mathbb{C P}^{n+1} ; R j_{*} \mathcal{L}\right) & \cong H^{2 n+2-i}(\mathcal{U} ; \mathcal{L}) \\
& \cong \begin{cases}0 & i<n+1, \\
\Gamma^{\mu} \oplus \overline{H_{n}\left(\mathcal{U}^{c}\right)} & i=n+1, \\
\overline{H_{2 n+1-i}\left(\mathcal{U}^{c}\right)} & i>n+1\end{cases}
\end{aligned}
$$

Recall that $\delta_{i}(t)$ denotes the Alexander polynomial associated to the Alexander module $H_{i}\left(\mathcal{U}^{c}\right)(i \leq n)$. Let $r_{i}(t)$ be the Alexander polynomial of the torsion $\Gamma$-module $H^{2 n+1-i}\left(\mathbb{C P}^{n+1} ; \mathcal{R}^{\bullet}\right)$. The above proposition yields the following relationship between the polynomials $r_{i}$ and $\delta_{i}$ :

Corollary 4.2 We have

$$
r_{i}(t)= \begin{cases}\frac{\delta_{i}(t)}{\delta_{2 n-i}(t)} & i<n\end{cases}
$$

and $\delta_{n}(t) \cdot \overline{\delta_{n}(t)}$ divides $r_{n}(t)$.

Set

$$
\varphi(t)=\frac{r_{n}(t)}{\delta_{n}(t) \cdot \overline{\delta_{n}(t)}} .
$$

Let $F_{h}$ denote as before the Milnor fibre associated to the polynomial $h=f_{d}$, the top-degree part of the polynomial $f$. Let $h_{i}(t)$ be the Alexander polynomial associated to $H_{i}\left(F_{h}\right)$. Then it was shown in [25, Theorem 4.7] that $h_{i}(t)=\delta_{i}(t)$ for $i<n$, and $\delta_{n}(t)$ divides $h_{n}(t)$. Set

$$
\varphi_{1}(t)=\frac{h_{n}(t)}{\delta_{n}(t)}
$$


Similarly, we let $\psi_{i}(t)$ denote the Alexander polynomial associated to the torsion $\Gamma$-module $H_{c}^{2 n+1-i}\left(F_{0} ; \psi_{f}^{S} \mathbb{Q}\right)$. It was shown in [23, Theorem 1.1] that $\psi_{i}(t)=\delta_{i}(t)$ for $i<n$, and $\delta_{n}(t)$ divides $\psi_{n}(t)$. Set

$$
\varphi_{2}(t)=\frac{\psi_{n}(t)}{\delta_{n}(t)}
$$

As can be seen from their definitions, the polynomials $\varphi_{1}(t)$ and $\varphi_{2}(t)$ can be regarded as error estimates for the Alexander polynomial $\delta_{n}(t)$. The above polynomial invariants are related by the following result:

Theorem 4.3 Assume that the polynomial $f: \mathbb{C}^{n+1} \rightarrow \mathbb{C}$ is transversal at infinity. With the above notations, we have the equalities

$$
\begin{aligned}
r_{n}(t) & =\overline{h_{n}(t)} \cdot \psi_{n}(t)=h_{n}(t) \cdot \overline{\psi_{n}(t)}, \\
\varphi(t) & =\overline{\varphi_{1}(t)} \cdot \varphi_{2}(t)=\varphi_{1}(t) \cdot \overline{\varphi_{2}(t)} .
\end{aligned}
$$

Remark 4.4 Since the polynomials $h_{i}(t), \delta_{i}(t)$ and $\psi_{i}(t)$ are products of cyclotomic polynomials (eg see [23]), the involution operation $\bar{*}$ keeps these polynomials unchanged.

In the course of proving Theorem 4.3, we need the following technical result:

Lemma 4.5 We have the $\Gamma$-module isomorphisms, for all $i$,

$$
\begin{gathered}
H_{c}^{2 n+1-i}\left(H \backslash V \cap H ; \mathcal{R}^{\bullet}\right) \cong H_{i}\left(F_{h}\right), \\
H^{2 n+1-i}\left(H ; \mathcal{R}^{\bullet}\right) \cong H_{i}\left(F_{h}, \partial F_{h}\right), \\
H^{2 n+1-i}\left(V \cap H ; \mathcal{R}^{\bullet}\right) \cong H_{i-1}\left(\partial F_{h}\right) .
\end{gathered}
$$

Proof Choose coordinates $\left[x_{0}, \ldots, x_{n+1}\right]$ for $\mathbb{C P}^{n+1}$, so that $H=\left\{x_{0}=0\right\}$ is the hyperplane at infinity. Then $\mathbb{O}=[0, \ldots, 0,1]$ corresponds to the origin in $\mathbb{C}^{n+1}$. Define

$$
\alpha: \mathbb{C P}^{n+1} \rightarrow \mathbb{R}_{+}, \quad \alpha:=\frac{\left|x_{0}\right|^{2}}{\sum_{i=0}^{n+1}\left|x_{i}\right|^{2}} .
$$

Note that $\alpha$ is a well-defined, real analytic and proper function satisfying

$$
0 \leq \alpha \leq 1, \quad \alpha^{-1}(0)=H \quad \text { and } \quad \alpha^{-1}(1)=\mathbb{O} .
$$

Since $\alpha$ has only finitely many critical values, there exists $\eta$ sufficiently small that the interval $(0, \eta]$ contains no critical values. Set

$$
U_{\eta}=\alpha^{-1}[0, \eta) .
$$


Then $U_{\eta}$ is a tubular neighbourhood of $H$ in $\mathbb{C P}^{n+1}$, and note that $\mathbb{C P}^{n+1} \backslash U_{\eta}$ is a closed large ball of radius $(1-\eta) / \eta$ in $\mathbb{C}^{n+1}$. Set

$$
U_{\eta}^{*}=\alpha^{-1}(0, \eta)=U_{\eta} \backslash H .
$$

Let us now consider the commutative diagram of inclusions

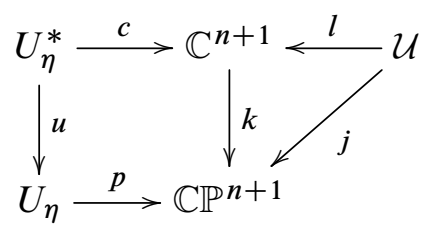

and restrict the distinguished triangle (3-6) over $U_{\eta}$. We get a triangle

$$
p^{*} R k_{*} l_{!} \mathcal{L} \rightarrow p^{*} R k_{*} R l_{*} \mathcal{L} \rightarrow p^{*} v_{!}\left(\left.\mathcal{R}^{\bullet}\right|_{V}\right) \stackrel{[1]}{\rightarrow},
$$

where $v$ denotes as before the inclusion of $V$ in $\mathbb{C P}^{n+1}$. Let us first give geometric interpretations to all $\Gamma$-modules appearing in the hypercohomology long exact sequence associated to (4-9). First note that

$$
\begin{aligned}
p^{*} R k_{*} l_{!} \mathcal{L}[2 n+2] & \stackrel{(1)}{\cong} R u_{*} c^{*} l ! \mathcal{L}[2 n+2] \\
& \stackrel{(2)}{\cong} R u_{*} c^{*} k^{*} k_{!} l_{!} \mathcal{L}[2 n+2] \\
& \cong R u_{*} c^{*} k^{*} j ! \mathcal{L}[2 n+2] \\
& \stackrel{(3)}{\cong} R u_{*} u^{*} p^{*} I C_{\bar{m}}\left(\mathbb{C} P^{n+1}, \mathcal{L}\right) \\
& \cong R u_{*} I C_{\bar{m}}\left(U_{\eta}^{*}, \mathcal{L}\right),
\end{aligned}
$$

where (1) follows from the base change isomorphism $p^{!} R k_{*}=R u_{*} c^{!}$(together with $p^{!}=p^{*}$ and $c^{!}=c^{*}$, as $p$ and $c$ are both open inclusions), for (2) we use the known identity $k^{*} k_{\text {! }} \cong \mathrm{id}$, and (3) follows from Theorem 2.4.

Set

$$
S_{\infty}=\alpha^{-1}\left(\eta^{\prime}\right)
$$

for $0<\eta^{\prime}<\eta$. Then we get, as in [25, Theorem 4.7],

$$
\begin{aligned}
H^{2 n+1-i}\left(U_{\eta} ; p^{*} R k_{*} R l_{!} \mathcal{L}\right) & \cong H^{-i-1}\left(U_{\eta}^{*} ; I C_{\bar{m}}\left(U_{\eta}^{*}, \mathcal{L}\right)\right) \\
& \stackrel{(1)}{\cong} H^{-i-1}\left(S_{\infty} ;\left.I C_{\bar{m}}\left(U_{\eta}^{*}, \mathcal{L}\right)\right|_{S_{\infty}}\right) \\
& \cong H_{i}\left(S_{\infty} \backslash S_{\infty} \cap V ; \mathcal{L}\right) \\
& \stackrel{(2)}{\cong} H_{i}\left(F_{h}\right)
\end{aligned}
$$


where (1) follows from [17, Lemma 8.4.7(c)], and (2) follows from the fact that $V$ intersects $H$ transversally. In fact, the corresponding infinite cyclic cover of $S_{\infty} \backslash S_{\infty} \cap V$ is homotopy equivalent to $F_{h}$; see [25, Proposition 4.9]. Also note that $\left.\left.I C_{\bar{m}}\left(U_{\eta}^{*}, \mathcal{L}\right)\right|_{S_{\infty}} \cong I C_{\bar{m}}\left(\mathbb{C P}^{n+1}, \mathcal{L}\right)\right|_{S_{\infty}}$. Similarly, by using Theorem 2.4 and duality, we have

$$
H^{2 n+1-i}\left(U_{\eta} ; p^{*} R k_{*} R l_{*} \mathcal{L}\right) \cong H^{-i-1}\left(S_{\infty} ;\left.I C_{\bar{l}}\left(\mathbb{C P}^{n+1}, \mathcal{L}\right)\right|_{S_{\infty}}\right) \cong H_{i}\left(F_{h}, \partial F_{h}\right) .
$$

So, by comparing the hypercohomology long exact sequence associated to (4-9) with the homology long exact sequence induced by the natural inclusion $\partial F_{h} \rightarrow F_{h}$ and using the above calculations, we get the $\Gamma$-module isomorphism

$$
H^{2 n+1-i}\left(S_{\infty} ;\left.\mathcal{R} \bullet\right|_{\infty}\right) \cong H_{i-1}\left(\partial F_{h}\right)
$$

Recall that the triangle (3-6) was obtained from (3-5) by a base change isomorphism, so the associated hypercohomology long exact sequences for the restrictions of these triangles over $U_{\eta}$ coincide. Note that [17, Lemma 8.4.7(a)] shows that, for any $\mathcal{F} \in D_{c}^{b}\left(\mathbb{C P}^{n+1}\right)$, there is an isomorphism

$$
H^{*}\left(U_{\eta} ; \mathcal{F}^{*}\right) \cong H^{*}\left(H ; \mathcal{F}^{*}\right)
$$

So, by restricting (3-5) over $H$ we get the same hypercohomology long exact sequence as for restricting (3-5) and (3-6) over $U_{\eta}$. Let $i_{h v}$ be the inclusion of $H \cap V$ into $H$. By using the proper base change isomorphism [9, Theorem 2.3.26] for the diagram

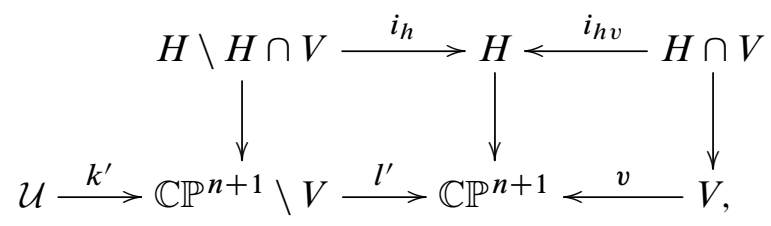

we have (using the notations of Theorem 3.1)

$$
\begin{gathered}
\left.\left(R l_{!}^{\prime} R k_{*}^{\prime} \mathcal{L}\right)\right|_{H}=R i_{h !}\left(\left.\left(R k_{*}^{\prime} \mathcal{L}\right)\right|_{H \backslash H \cap V}\right)=R i_{h !}\left(\left.\mathcal{R}^{\bullet}\right|_{H \backslash H \cap V}\right), \\
\left.\left(R v_{!}\left(\left.\mathcal{R}^{\bullet}\right|_{V}\right)\right)\right|_{H}=R i_{h v !}\left(\left.\mathcal{R}^{\bullet}\right|_{H \cap V}\right) .
\end{gathered}
$$

So, the hypercohomology long exact sequence associated to the restriction of the triangle (3-5) over $H$ becomes

$\cdots \rightarrow H_{c}^{2 n+1-i}\left(H \backslash V \cap H ; \mathcal{R}^{\bullet}\right) \rightarrow H^{2 n+1-i}\left(H ; \mathcal{R}^{\bullet}\right) \rightarrow H^{2 n+1-i}\left(V \cap H ; \mathcal{R}^{\bullet}\right) \rightarrow \cdots$ 
Therefore, by the above calculations for the restriction of (3-6) over $U_{\eta}$, we get the $\Gamma$-module isomorphisms

$$
\begin{aligned}
H_{c}^{2 n+1-i}\left(H \backslash V \cap H ; \mathcal{R}^{\bullet}\right) & \cong H_{i}\left(F_{h}\right), \\
H^{2 n+1-i}\left(H ; \mathcal{R}^{\bullet}\right) & \cong H_{i}\left(F_{h}, \partial F_{h}\right), \\
H^{2 n+1-i}\left(V \cap H ; \mathcal{R}^{\bullet}\right) & \cong H_{i-1}\left(\partial F_{h}\right)
\end{aligned}
$$

for all $i$.

Proof of Theorem 4.3 Let us consider the long exact sequence of hypercohomology with compact supports for the peripheral complex $\mathcal{R}^{\bullet}$ with respect to the inclusions

$$
F_{0} \hookrightarrow V \cup H \hookleftarrow H .
$$

By using duality and [25, Theorem 4.7], we have that

$$
H_{i}\left(F_{h}, \partial F_{h}\right) \cong \overline{H_{2 n-i}\left(F_{h}\right)} \cong \overline{H_{2 n-i}\left(\mathcal{U}^{c}\right)}
$$

for $i>n$. On the other hand, [23, Theorem 1.1] yields that

$$
H_{c}^{2 n+1-i}\left(F_{0} ; \psi_{f}^{S} \mathbb{Q}\right) \cong H_{i}\left(\mathcal{U}^{c}\right)
$$

for $i<n$. By using Proposition 4.1 and vanishing results for perverse sheaves on affine spaces, we obtain the $\Gamma$-module isomorphisms

$$
\begin{aligned}
H_{c}^{2 n+1-i}\left(F_{0} ; \psi_{f}^{S} \mathbb{Q}\right) \cong H^{2 n+1-i}\left(V \cup H ; \mathcal{R}^{\bullet}\right) & & \text { for } i<n, \\
H^{2 n+1-i}\left(V \cup H ; \mathcal{R}^{\bullet}\right) \cong H^{2 n+1-i}\left(H ; \mathcal{R}^{\bullet}\right) \cong H_{i}\left(F_{h}, \partial F_{h}\right) & & \text { for } i>n
\end{aligned}
$$

and a short exact sequence for $i=n$,

$$
0 \rightarrow H_{c}^{n+1}\left(F_{0} ; \psi_{f}^{S} \mathbb{Q}\right) \rightarrow H^{n+1}\left(V \cup H ; \mathcal{R}^{\bullet}\right) \rightarrow H^{n+1}\left(H ; \mathcal{R}^{\bullet}\right) \rightarrow 0 .
$$

Similarly, for the inclusions $(H \backslash V \cap H) \hookrightarrow V \cup H \hookleftarrow V$, there is a short exact sequence

$$
0 \rightarrow H_{c}^{n+1}\left(H \backslash V \cap H ; \mathcal{R}^{\bullet}\right) \rightarrow H^{n+1}\left(V \cup H ; \mathcal{R}^{\bullet}\right) \rightarrow H^{n+1}\left(V ; \mathcal{R}^{\bullet}\right) \rightarrow 0 .
$$

By using (4-7) and duality, the Alexander polynomial associated to $H^{n+1}\left(H ; \mathcal{R}^{\bullet}\right)$ is $\overline{h_{n}(t)}$. Moreover, since

$$
\mathcal{D}\left(\psi_{f}^{S} \mathbb{Q}\right)=\left(\psi_{f}^{S} \mathbb{Q}\right)^{\mathrm{op}}[2 n+1],
$$

we have by Theorem 3.1 that

$$
\begin{aligned}
H^{n+1}\left(V ; \mathcal{R}^{\bullet}\right) \cong H^{n+1}\left(V ; i_{v *} \psi_{f}^{S} \mathbb{Q}\right) & \cong H^{n+1}\left(F_{0} ; \psi_{f}^{S} \mathbb{Q}\right) \\
& \cong H_{c}^{n+1}\left(F_{0} ;\left(\psi_{f}^{S} \mathbb{Q}\right)^{\mathrm{op}}\right)
\end{aligned}
$$


where the last isomorphism follows from duality and the universal coefficient theorem. So, the Alexander polynomial associated to $H^{n+1}\left(V ; \mathcal{R}^{\bullet}\right)$ is $\overline{\psi_{n}(t)}$.

Since $\mathcal{R}^{\bullet}$ is supported on $V \cup H$, the result follows now by using the multiplicativity of the Alexander polynomials associated to the short exact sequences (4-16) and (4-17).

We conclude this section with the following degree estimate:

Proposition 4.6 Assume that the polynomial $f: \mathbb{C}^{n+1} \rightarrow \mathbb{C}$ is transversal at infinity.

(a) We have the degree estimates

$$
\operatorname{deg} \varphi_{2} \leq \operatorname{deg} \varphi_{1} \leq d \cdot \mu,
$$

hence

$$
\operatorname{deg} \varphi \leq 2 d \cdot \mu,
$$

where $\mu=|\chi(\mathcal{U})|$ and $d$ is the degree of $f$.

(b) If $F$ denotes the generic fibre of $f$, then $F_{h}$ and $F$ have isomorphic $\mathbb{Z}$ homology groups.

(c) Let $\tilde{f}$ be the homogenization of $f$, with corresponding Milnor fibre $\widetilde{F}=$ $\{\tilde{f}=1\}$. Then, if $\mu=0$, the spaces $\mathcal{U}^{c}, F, F_{h}$ and $\widetilde{F}$ are all homotopy equivalent to each other.

Proof (a) Let $\widetilde{F}_{t}$ be the Milnor fibre of $\tilde{f}$ defined by $\{\tilde{f}=t\}$ for small enough $t \in \mathbb{C}^{*}$. Clearly, $\widetilde{F}_{t}$ is homotopy equivalent to $\widetilde{F}$. Without loss of generality, $t$ can be chosen so that $F_{t}=f^{-1}(t)$ is the generic fibre of $f$, hence $F_{t}$ is smooth. Since $V$ intersects $H$ transversally, the hyperplane $\left\{x_{0}=0\right\}$ in $\mathbb{C}^{n+2}$ and its parallel hyperplane $\left\{x_{0}=1\right\}$ are both generic for $\widetilde{F}_{t}$ in the sense of [13]. It follows that, up to homotopy, $\widetilde{F}_{t}$ is obtained from either the Milnor fibre $F_{h}$ of $h=f_{d}$, or from the generic fibre $F_{t}$, by attaching $d \cdot \mu$ cells of dimension $n+1$ [13, Proposition 9]. On the other hand, [23, Corollary 6.5] shows that there exists a natural map from $\mathcal{U}^{c}$ to $\widetilde{F}$, which induces an $(n+1)$-homotopy equivalence and, in particular, we have that $H_{n}\left(\mathcal{U}^{c}\right) \cong H_{n}(\tilde{F})$. These two facts together yield that $\operatorname{deg} \varphi_{1} \leq d \cdot \mu$. Note also that by [23, Theorem 1.2] we have that $\operatorname{dim} H_{c}^{n}\left(F_{0}, \psi_{f} \mathbb{Q}\right) \leq \operatorname{dim} H_{n}\left(F_{t}\right)$. Therefore, $\operatorname{deg} \varphi_{2} \leq \operatorname{deg} \varphi_{1} \leq d \cdot \mu$, so $\operatorname{deg} \varphi=\operatorname{deg} \varphi_{1}+\operatorname{deg} \varphi_{2} \leq 2 d \cdot \mu$.

(b) The above homotopy argument yields the following isomorphisms for $i \leq n-1$ :

$$
H_{i}\left(F_{h}, \mathbb{Z}\right) \cong H_{i}(\widetilde{F}, \mathbb{Z}) \cong H_{i}(F, \mathbb{Z}) .
$$

Since $F_{h}$ and $F$ are $n$-dimensional affine varieties, both of them have the homotopy type of a finite $n$-dimensional $\mathrm{CW}$ complex. So $H_{n}\left(F_{h}, \mathbb{Z}\right)$ and $H_{n}(F, \mathbb{Z})$ are free 
abelian groups, and it remains to show that they have the same rank. This is indeed true, since the above discussion shows that $\chi\left(F_{h}\right)=\chi(F)$.

(c) If $\mu=0$, then $F_{h}$ and $F$ are homotopy equivalent to $\widetilde{F}$. In particular, the natural map from $\mathcal{U}^{c}$ to $\widetilde{F}$ induces isomorphisms on all homology groups with $\mathbb{Z}$-coefficients. Since this map is already an $(n+1)$-homotopy equivalence, it follows by Hurewicz's theorem that $\mathcal{U}^{c}$ is homotopy equivalent to $\widetilde{F}$.

Remark 4.7 In fact, we proved the equalities

$$
\begin{gathered}
d \cdot \mu-\operatorname{deg} \varphi_{1}=\operatorname{dim} H_{n+1}(\tilde{F}), \\
\operatorname{dim} H_{n}(F)-\operatorname{dim} H_{c}^{n}\left(F_{0}, \psi_{f} \mathbb{Q}\right)=\operatorname{deg} \varphi_{1}-\operatorname{deg} \varphi_{2} .
\end{gathered}
$$

\section{Reidemeister torsion and Alexander polynomials}

\subsection{Reidemeister torsion of chain complexes}

In this section, we recall the definition and main results about the Reidemeister torsion, for more details see Kirk and Livingston [18] and Cogolludo and Florens [5].

Let $C_{*}$ be a finite chain complex

$$
C_{*}=C_{n} \stackrel{\partial}{\longrightarrow} \cdots \stackrel{\partial}{\longrightarrow} C_{0}
$$

with $C_{i}$ finite-dimensional $\mathbb{F}$-vector spaces and $\partial \circ \partial=0$. Choose a basis $c_{i}$ for $C_{i}$, $\overline{h_{i}}$ a basis for the homology $H_{i}\left(C_{*}\right)$ and $h_{i}$ a lift of $\overline{h_{i}}$ to $C_{i}$. Let $b_{i}$ be a basis for $B_{i}:=\operatorname{Image}\left(\partial: C_{i+1} \rightarrow C_{i}\right)$ and $\hat{b}_{i}$ a lift of $b_{i}$ in $C_{i+1}$. If $Z_{i}$ denotes the $i$-cycles, by using the inclusions $B_{i} \subseteq Z_{i} \subseteq C_{i}$ together with the isomorphisms $Z_{i} / B_{i} \cong H_{i}\left(C_{*}\right)$ and $C_{i} / Z_{i} \cong B_{i-1}$ it follows that $b_{i} h_{i} \hat{b}_{i-1}$ is a basis of $C_{i}$. Denote by $[a \mid b]$ the determinant of the transition matrix from the basis $a$ to the basis $b$.

Definition 5.1 [27] The torsion of $\left(C_{*} ; c, h\right)$ is defined as

$$
\tau\left(C_{*} ; c, h\right)=\prod_{i=0}^{n}\left[b_{i} h_{i} \widehat{b}_{i-1} \mid c_{i}\right]^{(-1)^{i}} \in \mathbb{F}^{*} /\{ \pm 1\} .
$$

The torsion does not depend on the choice of basis $b$ and its lifts. It depends on the choice of $c$ and $h$ as follows:

$$
\tau\left(C_{*} ; c^{\prime}, h^{\prime}\right)=\tau\left(C_{*} ; c, h\right) \prod_{i}\left(\frac{\left[h_{i}^{\prime} \mid h_{i}\right]}{\left[c_{i}^{\prime} \mid c_{i}\right]}\right)^{(-1)^{i}} .
$$




\subsection{Torsion and Alexander polynomials}

Let $X$ be a finite connected CW complex, with $\pi=\pi_{1}(X)$. Fix an epimorphism $\rho: \pi \rightarrow \mathbb{Z}$ and note that $\rho$ extends naturally to an epimorphism of algebras $\mathbb{Z}[\pi] \rightarrow \mathbb{Z}[\mathbb{Z}]$, which we also denote by $\rho$. We identify $\mathbb{Z}[\mathbb{Z}]$ with the Laurent polynomial ring $\mathbb{Z}\left[t, t^{-1}\right]$. If $\tilde{X} \rightarrow X$ is the universal cover of $X$, then the cellular chain complex $C_{*}(\tilde{X} ; \mathbb{Q})$ is a $\mathbb{Q}[\pi]$-module, freely generated by the lifts of the cells of $X$. Consider the chain complex of the pair $(X, \rho)$ defined as the complex of $\mathbb{Q}\left[t, t^{-1}\right]$-modules

$$
C_{*}^{\rho}\left(X ; \mathbb{Q}\left[t, t^{-1}\right]\right):=\mathbb{Q}\left[t, t^{-1}\right] \otimes_{\mathbb{Q}[\pi]} C_{*}(\tilde{X} ; \mathbb{Q}) .
$$

Let $X^{c}$ denote the infinite cyclic cover defined by the kernel of $\rho$. Then, under the action of the deck group $\mathbb{Z}$, the chain complex $C_{*}\left(X^{c}, \mathbb{Q}\right)$ becomes a complex of $\Gamma:=\mathbb{Q}\left[t, t^{-1}\right]$-modules that is canonically isomorphic to $C_{*}^{\rho}\left(X ; \mathbb{Q}\left[t, t^{-1}\right]\right)$.

Denote by $\mathbb{Q}(t)$ the fraction field of $\mathbb{Q}\left[t, t^{-1}\right]$ and define

$$
C_{*}^{\rho}(X, \mathbb{Q}(t))=C_{*}\left(X^{c}, \mathbb{Q}\right) \otimes_{\mathbb{Q}\left[t, t^{-1}\right]} \mathbb{Q}(t) .
$$

The $i^{\text {th }}$ homology of $(X, \rho)$ (also called the $i^{\text {th }}$ Alexander module) is defined to be the $\mathbb{Q}\left[t, t^{-1}\right]$-module

$$
H_{i}^{\rho}\left(X, \mathbb{Q}\left[t, t^{-1}\right]\right):=H_{i}\left(C_{*}^{\rho}\left(X ; \mathbb{Q}\left[t, t^{-1}\right]\right)\right) \cong H_{i}\left(X^{c}, \mathbb{Q}\right),
$$

and we extend the definition to $H_{i}^{\rho}(X, \mathbb{Q}(t)):=H_{i}\left(C_{*}^{\rho}(X, \mathbb{Q}(t))\right)$. Since $\mathbb{Q}\left[t, t^{-1}\right]$ is a principal ideal domain and $\mathbb{Q}(t)$ is flat over $\mathbb{Q}\left[t, t^{-1}\right]$, it follows that

$$
H_{i}^{\rho}(X, \mathbb{Q}(t))=H_{i}^{\rho}\left(X, \mathbb{Q}\left[t, t^{-1}\right]\right) \otimes \mathbb{Q}(t) .
$$

Note that the complex $C_{*}^{\rho}(X ; \mathbb{Q}(t))$ is $\mathbb{Q}(t)$-acyclic if $H_{i}^{\rho}\left(X, \mathbb{Q}\left[t, t^{-1}\right]\right)$ is a torsion $\Gamma$-module for all $i$.

We now define the Reidemeister torsion for the pair $(X, \rho)$. For this, we first note that the complex $C_{*}^{\rho}(X ; \mathbb{Q}(t))$ is based by construction.

Definition 5.2 Fix a basis for the homology $H_{*}^{\rho}(X, \mathbb{Q}(t))$. The Reidemeister torsion of $(X, \rho)$ with respect to this basis is defined as

$$
\tau_{\rho}(X)=\tau\left(C_{*}^{\rho}(X, \mathbb{Q}(t))\right) \in \mathbb{Q}(t)^{*} .
$$

We next indicate a basis-free definition for the Reidemeister torsion. Since $\mathbb{Q}\left[t, t^{-1}\right]$ is a PID, any $\mathbb{Q}\left[t, t^{-1}\right]$-module $M$ has a decomposition into a direct sum of cyclic modules. Recall that the order of $M$ is defined as the product of the generators of the torsion part. If the module $M$ is free, the order is 1 by convention. 
Definition 5.3 The $i^{\text {th }}$ Alexander polynomial $\delta_{i}^{\rho}(X)$ of $(X, \rho)$ is defined to be the order of the $\mathbb{Q}\left[t, t^{-1}\right]$-module $H_{i}^{\rho}\left(X, \mathbb{Q}\left[t, t^{-1}\right]\right)$.

The torsion of $(X, \rho)$ can be computed in terms of the Alexander polynomials as follows:

Theorem $5.4\left[18\right.$, Theorem 3.4] Let $\tau_{\rho}(X)$ be the torsion of $(X, \rho)$ with respect to some basis in homology. Then, up to multiplication by $c t^{k}\left(c \in \mathbb{Q}^{*}\right.$ and $\left.k \in \mathbb{Z}\right)$, we have that

$$
\tau_{\rho}(X)=\prod_{i} \frac{\delta_{2 i+1}^{\rho}(X)}{\delta_{2 i}^{\rho}(X)} .
$$

So we can regard the right-hand side of (5-3) as a basis-free definition of the Reidemeister torsion.

\subsection{Duality and intersection forms}

Let $X$ be a smooth compact $2 m$-dimensional manifold with boundary $\partial X$. Then it is known that $X$ has a PL structure and any two PL-triangulations have a common linear subdivision which is PL. Endow $X$ with the CW decomposition induced by one of these. Since $X$ is compact, the associated CW complex is finite. Note that $\partial X$ inherits the structure of a PL-manifold from $X$ and the triangulations of $X$ can be used to define the chain complex $C_{*}^{\rho}(X, \partial X, \mathbb{Q}(t))$. In this setting one can construct non-singular Poincaré duality pairings for $0 \leq i \leq 2 \mathrm{~m}$,

$$
H_{i}^{\rho}(X, \mathbb{Q}(t)) \times H_{2 m-i}^{\rho}(X, \partial X, \mathbb{Q}(t)) \rightarrow \mathbb{Q}(t) .
$$

The intersection form of $(X, \rho)$ is the sesquilinear form

$$
\phi^{\rho}: H_{m}^{\rho}(X, \mathbb{Q}(t)) \times H_{m}^{\rho}(X, \mathbb{Q}(t)) \rightarrow \mathbb{Q}(t)
$$

defined by the composition

$$
H_{m}^{\rho}(X, \mathbb{Q}(t)) \times H_{m}^{\rho}(X, \mathbb{Q}(t)) \rightarrow H_{m}^{\rho}(X, \mathbb{Q}(t)) \times H_{m}^{\rho}(X, \partial X, \mathbb{Q}(t)) \rightarrow \mathbb{Q}(t),
$$

where the first map is induced by inclusion and the second map is the pairing (5-4).

Let $\bar{*}$ denote the canonical involution on $\mathbb{Q}\left[t, t^{-1}\right]$. For each $i$ and for a fixed basis on $H_{i}^{\rho}(X, \mathbb{Q}(t))$, we choose the dual basis on $H_{2 m-i}^{\rho}(X, \partial X, \mathbb{Q}(t))$ given by (5-4). Then we have $[18 ; 26]$ that

$$
\tau_{\rho}(X, \partial X) \cdot \overline{\tau_{\rho}(X)}=1
$$

The following result will be useful later: 
Lemma $5.5[5 ; 18]$ For any $\left(X^{2 m}, \rho\right)$ as above such that $X$ has the homotopy type of an $m$-dimensional $C W$ complex and $C_{*}^{\rho}(\partial X, \mathbb{Q}(t))$ is acyclic,

$$
\tau_{\rho}(\partial X)=\tau_{\rho}(X) \cdot \overline{\tau_{\rho}(X)} \cdot\left[\operatorname{det}\left(\phi^{\rho}\right)\right]^{(-1)^{m}} .
$$

\section{Boundary manifold of a hypersurface complement}

In this section, we define the boundary manifold of a hypersurface complement and investigate its topology. We make use of the peripheral complex to study the linking number infinite cyclic cover of the boundary manifold. In particular, by using results from Section 3, we put mixed Hodge structures on the corresponding Alexander modules associated to the boundary manifold.

Choose coordinates $\left[x_{0}, \ldots, x_{n+1}\right]$ for $\mathbb{C P}^{n+1}$, and $H=\left\{x_{0}=0\right\}$. Define

$$
\theta: \mathbb{C P}^{n+1} \rightarrow \mathbb{R}_{+}, \quad \theta=\frac{|\tilde{f}|^{2}\left|x_{0}\right|^{2}}{\left(\sum_{i=0}^{n+1}\left|x_{i}\right|^{2}\right)^{d+1}},
$$

with $\tilde{f}$ denoting the homogenization of $f$ (so that $V$ is the zero set of $\tilde{f}$ in $\mathbb{C P}^{n+1}$ ). Note that $\theta$ is well-defined, it is real analytic and proper, and

$$
\theta^{-1}(0)=V \cup H \text {. }
$$

Since $\theta$ has only finitely many critical values, there exists a positive real number $\varepsilon$ sufficiently small that the interval $(0, \varepsilon]$ contains no critical values. Set

$$
\mathcal{U}_{0}=\theta^{-1}([\varepsilon,+\infty))
$$

So $\mathcal{U}_{0}$ is the complement of the regular tubular neighbourhood of $V \cup H$ in $\mathbb{C P}^{n+1}$.

Moreover, $\mathcal{U}_{0}$ is a manifold with boundary, homotopy equivalent to $\mathcal{U}$ (eg see Dimca [8, page 149]). Note that while $\mathcal{U}_{0}$ has the homotopy type of a finite $(n+1)$-dimensional $\mathrm{CW}$ complex, its boundary $\partial \mathcal{U}_{0}$ is a smooth, compact, real $(2 n+1)$-dimensional manifold. The inclusion $\partial \mathcal{U}_{0} \hookrightarrow \mathcal{U}_{0}$ is an $n$-homotopy equivalence [8, Proposition (5.2.31)], hence $\pi_{i}\left(\partial \mathcal{U}_{0}\right)=\pi_{i}\left(\mathcal{U}_{0}\right)$ for $i<n$, and we have an epimorphism $\pi_{n}\left(\partial \mathcal{U}_{0}\right) \rightarrow \pi_{n}\left(\mathcal{U}_{0}\right)$. Therefore

$$
\rho: \pi_{1}\left(\partial \mathcal{U}_{0}\right) \longrightarrow \pi_{1}\left(\mathcal{U}_{0}\right)=\pi_{1}(\mathcal{U}) \stackrel{f_{*}}{\longrightarrow} \pi_{1}\left(\mathbb{C}^{*}\right)=\mathbb{Z}
$$

is an epimorphism, which defines the infinite cyclic cover $\left(\partial \mathcal{U}_{0}\right)^{c}$ of $\partial \mathcal{U}_{0}$.

We refer to $\partial \mathcal{U}_{0}$ as the boundary manifold of the hypersurface complement $\mathcal{U}$. For a study of topological properties of such boundary manifolds, see Cohen and Suciu [6;7]. 
Proposition 6.1 We have the $\Gamma$-module isomorphisms

$$
H_{i}^{\rho}\left(\partial \mathcal{U}_{0}, \mathbb{Q}\left[t, t^{-1}\right]\right) \cong H_{i}\left(\left(\partial \mathcal{U}_{0}\right)^{c}\right) \cong H^{2 n+1-i}\left(V \cup H ; \mathcal{R}^{\bullet}\right) .
$$

In particular, $H_{i}\left(\left(\partial \mathcal{U}_{0}\right)^{c}\right)$ is a torsion $\Gamma$-module and $C_{*}^{\rho}\left(\partial \mathcal{U}_{0}, \mathbb{Q}(t)\right)$ is $\mathbb{Q}(t)$-acyclic. Moreover, the zeros of the Alexander polynomial associated to $H_{i}\left(\left(\partial \mathcal{U}_{0}\right)^{c}\right)$ are roots of unity for all $i$, and have order $d$ except for $i=n$. Finally, $H_{i}\left(\left(\partial \mathcal{U}_{0}\right)^{c}\right)$ is a semi-simple $\Gamma$-module for $i \neq n$.

Proof We have the isomorphisms of $\Gamma$-modules (see [25, Corollary 3.4, Lemma 3.5])

$$
\begin{aligned}
& I H_{i}^{\bar{m}}\left(\mathbb{C P}^{n+1}, \mathcal{L}\right) \cong H_{i}(\mathcal{U}, \mathcal{L}) \cong H_{i}\left(\mathcal{U}_{0}, \mathcal{L}\right) \cong H_{i}^{\rho}\left(U_{0}, \mathbb{Q}\left[t, t^{-1}\right]\right), \\
& I H_{i}^{\bar{l}}\left(\mathbb{C P}^{n+1} ; \mathcal{L}\right) \cong H_{i}^{\mathrm{BM}}(\mathcal{U} ; \mathcal{L}) \cong H_{i}^{\rho}\left(\mathcal{U}_{0}, \partial \mathcal{U}_{0} ; \mathbb{Q}\left[t, t^{-1}\right]\right),
\end{aligned}
$$

where $H_{*}^{\mathrm{BM}}$ denotes the Borel-Moore homology and the last isomorphism in (6-3) follows by Poincaré duality and homotopy equivalence. So, by comparing the homology exact sequence (with $\mathbb{Q}\left[t, t^{-1}\right]$-coefficients) of the pair $\left(\mathcal{U}_{0}, \partial \mathcal{U}_{0}\right)$ with the hypercohomology long exact sequence of the distinguished triangle defining the peripheral complex, we obtain the isomorphism of $\Gamma$-modules

$$
H_{i}^{\rho}\left(\partial \mathcal{U}_{0}, \mathbb{Q}\left[t, t^{-1}\right]\right) \cong H^{2 n+1-i}\left(\mathbb{C P}^{n+1} ; \mathcal{R}^{\bullet}\right) .
$$

And, since $\mathcal{R}^{\bullet}$ is supported on $V \cup H$, we obtain the isomorphisms in (6-1). Moreover, as $\mathcal{R}^{\bullet}$ is a $\Gamma$-torsion sheaf complex, (6-4) gives that $H_{i}\left(\left(\partial \mathcal{U}_{0}\right)^{c}\right) \cong H_{i}^{\rho}\left(\partial \mathcal{U}_{0}, \mathbb{Q}\left[t, t^{-1}\right]\right)$ is a $\Gamma$-torsion module. In particular, the chain complex $C_{*}^{\rho}\left(\partial \mathcal{U}_{0}, \mathbb{Q}(t)\right)$ is $\mathbb{Q}(t)$-acyclic. The zeros of the corresponding Alexander polynomials are roots of unity since this is the case for the Alexander polynomials associated to the modules $H^{2 n+1-i}\left(V \cup H ; \mathcal{R}^{\bullet}\right)$ (eg see [25]). The remaining claims follow from Theorem 2.2 and Proposition 4.1.

The following result is a direct consequence of Proposition 6.1 and Corollary 3.3.

Corollary 6.2 The Alexander modules $H_{i}\left(\left(\partial \mathcal{U}_{0}\right)^{c}\right)$ of the boundary manifold $\partial \mathcal{U}_{0}$ are endowed with mixed Hodge structures induced from the peripheral complex for all $i$. Moreover, for $i \neq n$, this mixed Hodge structure is compatible with the $\Gamma$-action, ie $t: H_{i}\left(\left(\partial \mathcal{U}_{0}\right)^{c}\right) \rightarrow H_{i}\left(\left(\partial \mathcal{U}_{0}\right)^{c}\right)$ is a mixed Hodge structure homomorphism for $i \neq n$.

Proof By Corollary 3.3, the peripheral complex $\mathcal{R}^{\bullet}$ underlies a (shifted) mixed Hodge module. Hence, the $\mathbb{Q}$-vector space isomorphism (underlying the $\Gamma$-module isomorphism of Proposition 6.1)

$$
H_{i}\left(\left(\partial \mathcal{U}_{0}\right)^{c}\right) \cong H^{2 n+1-i}\left(V \cup H ; \mathcal{R}^{\bullet}\right)
$$

defines a mixed Hodge structure on $H_{i}\left(\left(\partial \mathcal{U}_{0}\right)^{c}\right)$ for all $i$. 
In order to prove the second claim, note that by [23] the mixed Hodge structure on

$$
H_{c}^{2 n-i}\left(F_{0} ; \psi_{f} \mathbb{Q}\right) \cong H_{i}\left(F_{h}\right) \text { for } i<n
$$

is compatible with the $\Gamma$-action. Then the isomorphism (4-14) shows that the resulting mixed Hodge structure on $H_{i}\left(\left(\partial \mathcal{U}_{0}\right)^{c}\right)$ has the same property for $i<n$.

By Alexander duality on $H_{*}\left(F_{h}\right)$, the mixed Hodge structure on $H_{i}\left(F_{h}, \partial F_{h}\right)$ is compatible with the $\Gamma$-action for $i>n$. Then, by using the isomorphism (4-15), the resulting mixed Hodge structure on $H_{i}\left(\left(\partial \mathcal{U}_{0}\right)^{c}\right)$ satisfies the same property for $i>n$.

Our next result gives a geometric interpretation of the homology of the boundary manifold.

Let $g=x_{0} \tilde{f}$ be the homogeneous polynomial of degree $d+1$ whose zero locus is the divisor $V \cup H$. Consider the associated Milnor fibre $F_{g}=\{g=1\}$ and its boundary manifold $\partial F_{g}$. Then there exists a natural $(d+1)$-fold covering map (see [8, page 149])

$$
\partial F_{g} \rightarrow \partial \mathcal{U}_{0}
$$

Proposition 6.3 The covering map (6-6) induces isomorphisms of $\mathbb{Q}$-vector spaces

$$
H_{i}\left(\partial F_{g}\right) \cong H_{i}\left(\partial \mathcal{U}_{0}\right) \quad \text { for } i \neq n, n+1 .
$$

Moreover, if the complex numbers $\lambda_{\alpha}=\exp (2 \pi i \alpha /(d+1))$, with $\alpha=1,2, \ldots, d$, are not among the roots of $\psi_{n}(t)$, then the isomorphism (6-7) holds for all $i$. In particular, this is the case if $\mu=0$ (eg $f$ is homogeneous).

Proof Let $N(\lambda, i)$ be the number of direct summands in the $(t-\lambda)$-torsion part of $H_{i}\left(\left(\partial \mathcal{U}_{0}\right)^{c} ; \mathbb{C}\right)$, ie the number of the Jordan blocks with eigenvalue $\lambda$ for the automorphism on $H_{i}\left(\left(\partial \mathcal{U}_{0}\right)^{c} ; \mathbb{C}\right)$ induced by the $\Gamma$-action. Define a rank-one local system $\mathcal{L}_{\lambda}$ on $\partial \mathcal{U}_{0}$ by the composed map

$$
\pi_{1}\left(\partial \mathcal{U}_{0}\right) \stackrel{\rho}{\longrightarrow} \mathbb{Z} \longrightarrow \mathbb{C}^{*},
$$

where the last map is defined by $1_{\mathbb{Z}} \mapsto \lambda$. If $\lambda=1$, then $\mathcal{L}_{\lambda}=\mathbb{C}$. The Wang exact sequence

$\cdots \longrightarrow H_{i}\left(\left(\partial \mathcal{U}_{0}\right)^{c} ; \mathbb{C}\right) \stackrel{t-\lambda}{\longrightarrow} H_{i}\left(\left(\partial \mathcal{U}_{0}\right)^{c} ; \mathbb{C}\right) \longrightarrow H_{i}\left(\partial \mathcal{U}_{0} ; \mathcal{L}_{\lambda}\right) \longrightarrow H_{i-1}\left(\left(\partial \mathcal{U}_{0}\right)^{c} ; \mathbb{C}\right) \longrightarrow \cdots$

yields (eg see [12, Theorem 4.2]) that

$$
\operatorname{dim} H_{i}\left(\partial \mathcal{U}_{0} ; \mathcal{L}_{\lambda}\right)=N(\lambda, i)+N(\lambda, i-1)
$$


for all $i$. On the other hand, by using the $(d+1)$-fold covering map (6-6), we have that

$$
H_{i}\left(\partial F_{g} ; \mathbb{C}\right) \cong \bigoplus_{\lambda^{d+1}=1} H_{i}\left(\partial \mathcal{U}_{0} ; \mathcal{L}_{\lambda}\right)
$$

If $\lambda^{d} \neq 1$, then it follows from Proposition 6.1 that $N(\lambda, i)=0$ for $i \neq n$. (Note that $\operatorname{gcd}(d, d+1)=1$.) So, by using (6-8), we get the isomorphism (6-7) for $i \neq n, n+1$.

Moreover, if the complex numbers $\lambda_{\alpha}=\exp (2 \pi i \alpha /(d+1))$, with $\alpha=1,2, \ldots, d$, are not among the roots of $\psi_{n}(t)$, then the short exact sequence (4-16) shows that $N(\lambda, n)=0$ for $\lambda \in\left\{\lambda_{\alpha} \mid \alpha=1,2, \ldots, d\right\}$. So, in view of (6-8), the isomorphism (6-7) holds also for $i=n, n+1$. In particular, this is the case when $\mu=0$, since by [23, Proposition 4.2] it follows that $\psi_{n}(t)=h_{n}(t)$ has only roots of unity of order $d$.

Remark 6.4 The natural inclusion $\partial \mathcal{U}_{0} \hookrightarrow \mathcal{U}_{0}$ is an $n$-homotopy equivalence, and so is the inclusion $\partial F_{g} \hookrightarrow F_{g}$ (see [8, Proposition (3.2.4)]). Then Proposition 6.3 yields that $H_{i}\left(F_{g}\right) \cong H_{i}(\mathcal{U})$ for $i<n$ (compare with [10, Theorem 1.4]).

Example 6.5 Let $V \cup H$ be the hypersurface in $\mathbb{C P}^{n+1}$ defined by $g=x_{0} x_{1} \cdots x_{n+1}$. Then both $\partial F_{g}$ and $\partial \mathcal{U}_{0}$ are homotopy equivalent to $S^{n} \times\left(S^{1}\right)^{n+1}$ (see [8, Examples (5.2.29)]).

\section{Alexander polynomial estimates via Reidemeister torsion}

In this section, we refine the error estimates for Alexander polynomials given in Section 4 by making use of Reidemeister torsion and the intersection form.

Proposition 6.1 can be used to prove the following refinement of Theorem 4.3:

Theorem 7.1 Assume that the degree-d polynomial $f: \mathbb{C}^{n+1} \rightarrow \mathbb{C}$ is transversal at infinity. Let $\phi^{\rho}$ be the intersection form for $\left(\mathcal{U}_{0}, \partial \mathcal{U}_{0}\right)$ associated to $\rho$. Then, with the notations from Section 4, we have

$$
\begin{aligned}
\operatorname{det}\left(\phi^{\rho}\right) & =\varphi(t), \\
h_{n}(t) \cdot \psi_{n}(t) & =\delta_{n}^{2}(t) \cdot \operatorname{det}\left(\phi^{\rho}\right) .
\end{aligned}
$$

Moreover, $\operatorname{deg}\left(\operatorname{det}\left(\phi^{\rho}\right)\right)=\operatorname{deg} \varphi(t) \leq 2 d \cdot \mu$, where $\mu=|\chi(\mathcal{U})|$. 
Proof By Theorem 5.4, we have

$$
\tau_{\rho}\left(\mathcal{U}_{0}\right)=\tau_{\rho}(\mathcal{U})=\prod_{i=0}^{n} \delta_{i}(t)^{(-1)^{i+1}} .
$$

Since $\mathcal{U}_{0}$ has the homotopy type of a finite $(n+1)$-dimensional $\mathrm{CW}$ complex and the complex $C_{*}^{\rho}\left(\partial \mathcal{U}_{0}, \mathbb{Q}(t)\right)$ is $\mathbb{Q}(t)$-acyclic, Lemma 5.5 yields the Alexander polynomial identity

$$
\tau_{\rho}\left(\partial \mathcal{U}_{0}\right)=\prod_{i=0}^{n}\left\{\delta_{i}(t) \cdot \overline{\delta_{i}(t)}\right\}^{(-1)^{i+1}} \cdot\left[\operatorname{det}\left(\phi^{\rho}\right)\right]^{(-1)^{n+1}} .
$$

On the other hand, by using Theorem 5.4 and Proposition 6.1, we have

$$
\tau_{\rho}\left(\partial \mathcal{U}_{0}\right)=\prod_{i=0}^{2 n} r_{i}(t)^{(-1)^{i+1}}
$$

Recall that, by (4-3), the polynomials $r_{i}(t)$ and $\delta_{i}(t)$ are related by

$$
r_{i}(t)= \begin{cases}\delta_{i}(t) & i<n, \\ \delta_{2 n-i}(t) & i>n .\end{cases}
$$

So, by plugging (7-5) and (7-6) into formula (7-4), we obtain

$$
r_{n}(t)=\delta_{n}(t) \cdot \overline{\delta_{n}(t)} \cdot \operatorname{det}\left(\phi^{\rho}\right) .
$$

Therefore,

$$
\operatorname{det}\left(\phi^{\rho}\right)=\frac{r_{n}(t)}{\delta_{n}(t) \cdot \overline{\delta_{n}(t)}}=\varphi(t)
$$

and, by using Theorem 4.3 and Remark 4.4, we get the identity

$$
h_{n}(t) \cdot \psi_{n}(t)=\delta_{n}^{2}(t) \cdot \operatorname{det}\left(\phi^{\rho}\right) .
$$

The degree estimate $\operatorname{deg}\left(\operatorname{det}\left(\phi^{\rho}\right)\right)=\operatorname{deg} \varphi \leq 2 d \cdot \mu$ follows from Proposition 4.6.

Remark 7.2 Since $V$ intersects $H$ transversally, we can take the (closed) regular neighbourhood $N$ of $V \cup H$ to be the union of a regular neighbourhood $N(V)$ of $V$ with a tubular neighbourhood of the hyperplane at infinity (after rounding corners). Then

$$
\partial \mathcal{U}_{0}=\left(S_{R}^{2 n+1} \backslash\left(S_{R}^{2 n+1} \cap N^{\circ}(V)\right)\right) \cup\left(B_{R} \cap \partial N(V)\right),
$$

where $B_{R}$ is a closed large ball of radius $R$ in $\mathbb{C}^{n+1}$ with boundary sphere $S_{R}^{2 n+1}$. In [25, Proposition 4.9] it is shown that the infinite cyclic cover of $S_{R}^{2 n+1} \backslash\left(S_{R}^{2 n+1} \cap N(V)\right)$ is homotopy equivalent to the Milnor fibre $F_{h}$. Moreover, if $\left(B_{R} \cap \partial N(V)\right)^{c}$ denotes 
the corresponding infinite cyclic cover of $B_{R} \cap \partial N(V)$, it follows as in Lemma 4.5 that

$$
H_{i}\left(\left(B_{R} \cap \partial N(V)\right)^{c}\right) \cong H_{c}^{2 n+1-i}\left(F_{0}, \psi_{f}^{S} \mathbb{Q}\right)
$$

for all $i$. These two facts together give a geometric interpretation of Theorem 7.1, which is also consistent with the proof of [5, Theorem 5.6].

Remark 7.3 If $\mu=0$, then the chain complex $C_{*}^{\rho}\left(\mathcal{U}_{0}, \mathbb{Q}(t)\right)$ is $\mathbb{Q}(t)$-acyclic, thus the intersection pairing of Section 5.3 is trivial. In this case, we have $\operatorname{det}\left(\phi^{\rho}\right)=1$. This fact, coupled with the previous theorem, gives another proof of the result obtained in [23], asserting that

$$
\mu=0 \Longrightarrow \varphi=\varphi_{1}=\varphi_{2}=1
$$

Example 7.4 If $\mu=0$ (eg $f$ is homogeneous), then $\delta_{i}(t)=h_{i}(t)$ for all $i$. Then it is known (see $[8,(4.1 .21)])$ that

$$
\prod_{i=0}^{n} h_{i}(t)^{(-1)^{i+1}}=\left(t^{d}-1\right)^{-\chi\left(F_{h}\right) / d} \text {. }
$$

So,

$$
\tau_{\rho}\left(\partial \mathcal{U}_{0}\right)=\left(t^{d}-1\right)^{-2 \chi\left(F_{h}\right) / d} .
$$

As an application of Theorem 7.1, we have the following:

Corollary 7.5 Assume that the polynomial $f: \mathbb{C}^{n+1} \rightarrow \mathbb{C}$ is transversal at infinity and the hypersurface $F_{0}$ has only isolated singularities. Then

$$
(t-1)^{\mu+(-1)^{n+1}}\left(t^{d}-1\right)^{\xi} \prod_{p \in \operatorname{Sing}\left(F_{0}\right)} \Delta_{p}(t)=\delta_{n}(t)^{2} \cdot \operatorname{det}\left(\phi^{\rho}\right),
$$

where $\Delta_{p}(t)$ is the (top) local Alexander polynomial associated to the singular point $p \in \operatorname{Sing}\left(F_{0}\right)$ and $\xi=\left((d-1)^{n+1}+(-1)^{n}\right) / d$. In particular, $\operatorname{deg}\left(\operatorname{det}\left(\phi^{\rho}\right)\right)=\operatorname{deg} \varphi$ is even.

Proof We only need to compute the polynomials $\psi_{n}(t)$ and $h_{n}(t)$. For the case of isolated singularities, we have by [23, Section 5.2.1] that

$$
\psi_{n}(t)=(t-1)^{\mu} \prod_{p \in \operatorname{Sing}\left(F_{0}\right)} \Delta_{p}(t),
$$

while [8, Example (4.1.23)] provides another equality,

$$
h_{n}(t)=(t-1)^{(-1)^{n+1}}\left(t^{d}-1\right)^{\xi} \text {, }
$$


where $\xi=\left((d-1)^{n+1}+(-1)^{n}\right) / d$. Then (7-15) follows from Theorem 7.1.

Since $F_{0}$ has only isolated singularities, $V \cap H$ is a smooth hypersurface in $H$. Then $\left\{h+x_{0}^{d}=0\right\}$ is a smooth degree- $d$ hypersurface in $\mathbb{C P}^{n+1}$. By [8, Corollary (5.4.4)], $\chi\left(F_{0}, \psi_{f} \mathbb{Q}\right)+\chi(H \cap V)$ equals the Euler characteristic number of any smooth degree$d$ hypersurface in $\mathbb{C P}^{n+1}$, so, in particular,

$$
\chi\left(F_{0}, \psi_{f}\right)+\chi(H \cap V)=\chi\left(\left\{h+x_{0}^{d}=0\right\}\right) .
$$

Note also that

$$
\chi\left(F_{h}\right)+\chi(H \cap V)=\chi\left(\left\{h+x_{0}^{d}=0\right\}\right) .
$$

By the last two identities, we get $\chi\left(F_{h}\right)=\chi\left(F_{0}, \psi_{f} \mathbb{Q}\right)$, which shows that $\operatorname{deg} \varphi_{1}=$ $\operatorname{deg} \varphi_{2}$. So $\operatorname{deg} \varphi=\operatorname{deg} \varphi_{1}+\operatorname{deg} \varphi_{2} \equiv 0(\bmod 2)$.

Remark 7.6 (a) When $n=1$, Corollary 7.5 also follows from [5, Corollary 5.8].

(b) It would be interesting to see if the above property of (the degree of determinant of) the intersection pairing remains valid if $f$ has arbitrary singularities.

(c) For the case of isolated singularities, $\mu$ is given by the formula

$$
\mu=(d-1)^{n+1}-\sum_{p \in \operatorname{Sing}\left(F_{0}\right)} \mu_{p}
$$

where $\mu_{p}$ is the Milnor number of $f$ at $p$ (see [13]). Then the degree estimates of Theorem 7.1, together with Corollary 7.5, yield that

$$
2(d-1)^{n+1}=2 \operatorname{deg} \delta_{n}(t)+\operatorname{deg}\left(\operatorname{det}\left(\phi^{\rho}\right)\right) \leq 2 \operatorname{deg} \delta_{n}(t)+2 d \cdot \mu .
$$

Therefore,

$$
\operatorname{deg} \delta_{n}(t) \geq(d-1)^{n+1}-d \cdot \mu \text {. }
$$

In particular, we obtain a non-vanishing result for $H_{n}\left(\mathcal{U}^{c}\right)$ for small $\mu$. Such examples (with $\mu=1,2$ ) are given in [16].

Example 7.7 If $F_{0}$ is smooth, then $\delta_{n}(t)=1$ (see [21, Lemma 1.5]) and $\mu=$ $(d-1)^{n+1}$. So, by Corollary 7.5 , we conclude that

$$
\operatorname{det}\left(\phi^{\rho}\right)=(t-1)^{(d-1)^{n+1}+(-1)^{n+1}}\left(t^{d}-1\right)^{\xi},
$$

where $\xi=\left((d-1)^{n+1}+(-1)^{n}\right) / d$. 
Example 7.8 In relation to Remark 7.6(c), consider the hypersurface in $\mathbb{C P}^{3}$ defined by $V=\left\{x_{0} x_{1} x_{2}+x_{3}^{3}=0\right\}$. Then $V$ has only isolated singularities and it is known that $\mu=2$ for any generic hyperplane at infinity (see [16, Conjecture 20]). So, $\operatorname{deg} \delta_{2}(t) \geq(3-1)^{3}-3 \cdot 2=2$.

Example 7.9 Consider the hypersurface in $\mathbb{C P}^{2}$ defined by $V=\left\{x_{0} x_{1}^{3}+x_{1}^{4}+x_{2}^{4}=0\right\}$. The singular locus of $V$ is just one point, $p=[1,0,0]$. Let $H=\left\{x_{0}=0\right\}$ be the hyperplane at infinity. Note that $V \cap H$ is a smooth hypersurface in $H$, hence $V$ is transversal to $H$. The link pair of the point $p$ in $\left(\mathbb{C P}^{2}, V\right)$ is obtained by intersecting the affine variety $x_{1}^{3}+x_{1}^{4}+x_{2}^{4}=0$ in $\mathbb{C}^{2}$ with a small sphere about the origin. Since we work in a neighbourhood of the origin, by an analytic change of coordinates this is the same as the link pair of the origin in the variety $x_{1}^{3}+x_{2}^{4}=0$. The polynomial $x_{1}^{3}+x_{2}^{4}$ is weighted homogeneous with weighted degree 12 for the weight $(4,3)$, and the characteristic polynomial of the monodromy homeomorphism of the associated Milnor fibration is $\left(t^{4}-t^{2}+1\right)\left(t^{2}-t+1\right)$. So the link of the singular point is a rational homology sphere, which in turn yields that $F_{0}$ is a rational homology manifold. In particular, $\delta_{1}(1) \neq 0$ (eg see [23, Corollary 5.4]). Also note that the local Alexander polynomial of the link of the singularity has prime divisors, none of which divides $t^{4}-1$. Thus, they cannot be among the prime divisors of $\delta_{1}(t)$ (see [21]), hence $\delta_{1}(t)=1$. Equation (7-20) yields that $\mu=3$. By Corollary 7.5, we conclude that

$$
\operatorname{det}\left(\phi^{\rho}\right)=(t-1)^{4}\left(t^{4}-1\right)^{2}\left(t^{4}-t^{2}+1\right)\left(t^{2}-t+1\right) .
$$

Note also that $\operatorname{gcd}(12,5)=1$, so it follows from by Proposition 6.3 that $\partial F_{g}$ and $\partial \mathcal{U}_{0}$ have the same rational homology groups, where $g=x_{0}\left(x_{0} x_{1}^{3}+x_{1}^{4}+x_{2}^{4}\right)$.

\section{References}

[1] M Banagl, Topological invariants of stratified spaces, Springer, Berlin (2007) MR2286904

[2] J-L Brylinski, Transformations canoniques, dualité projective, théorie de Lefschetz, transformations de Fourier et sommes trigonométriques, from: "Géométrie et analyse microlocales", Astérisque 140-141, Soc. Math. France, Paris (1986) 3-134 MR864073

[3] N Budur, Bernstein-Saito ideals and local systems, Ann. Inst. Fourier 65 (2015) 549-603

[4] S E Cappell, J L Shaneson, Singular spaces, characteristic classes, and intersection homology, Ann. of Math. 134 (1991) 325-374 MR1127478

[5] J I Cogolludo Agustín, V Florens, Twisted Alexander polynomials of plane algebraic curves, J. Lond. Math. Soc. 76 (2007) 105-121 MR2351611 
[6] D C Cohen, A I Suciu, Boundary manifolds of projective hypersurfaces, Adv. Math. 206 (2006) 538-566 MR2263714

[7] D C Cohen, A I Suciu, The boundary manifold of a complex line arrangement, from: "Groups, homotopy and configuration spaces", (N Iwase, T Kohno, R Levi, D Tamaki, J Wu, editors), Geom. Topol. Monogr. 13 (2008) 105-146 MR2508203

[8] A Dimca, Singularities and topology of hypersurfaces, Springer, New York (1992) MR1194180

[9] A Dimca, Sheaves in topology, Springer, Berlin (2004) MR2050072

[10] A Dimca, A Libgober, Regular functions transversal at infinity, Tohoku Math. J. 58 (2006) 549-564 MR2297199

[11] A Dimca, L Maxim, Multivariable Alexander invariants of hypersurface complements, Trans. Amer. Math. Soc. 359 (2007) 3505-3528 MR2299465

[12] A Dimca, A Némethi, Hypersurface complements, Alexander modules and monodromy, from: "Real and complex singularities", (T Gaffney, M A S Ruas, editors), Contemp. Math. 354, Amer. Math. Soc. (2004) 19-43 MR2087802

[13] A Dimca, S Papadima, Hypersurface complements, Milnor fibers and higher homotopy groups of arrangments, Ann. of Math. 158 (2003) 473-507 MR2018927

[14] A H Durfee, Neighborhoods of algebraic sets, Trans. Amer. Math. Soc. 276 (1983) 517-530 MR688959

[15] M Goresky, R MacPherson, Intersection homology, II, Invent. Math. 72 (1983) 77 129 MR696691

[16] J Huh, Milnor numbers of projective hypersurfaces with isolated singularities, Duke Math. J. 163 (2014) 1525-1548 MR3210967

[17] M Kashiwara, P Schapira, Sheaves on manifolds, Grundl. Math. Wissen. 292, Springer, Berlin (1990) MR1074006

[18] P Kirk, C Livingston, Twisted Alexander invariants, Reidemeister torsion, and CassonGordon invariants, Topology 38 (1999) 635-661 MR1670420

[19] A Libgober, Alexander polynomial of plane algebraic curves and cyclic multiple planes, Duke Math. J. 49 (1982) 833-851 MR683005

[20] A Libgober, Alexander invariants of plane algebraic curves, from: "Singularities, II", (P Orlik, editor), Proc. Sympos. Pure Math. 40, Amer. Math. Soc. (1983) 135-143 MR713242

[21] A Libgober, Homotopy groups of the complements to singular hypersurfaces, II, Ann. of Math. 139 (1994) 117-144 MR1259366

[22] X S Lin, Representations of knot groups and twisted Alexander polynomials, Acta Math. Sin. (Engl. Ser.) 17 (2001) 361-380 MR1852950 
[23] Y Liu, Nearby cycles and Alexander modules of hypersurface complements arXiv: 1405.2343

[24] D Massey, Notes on perverse sheaves and vanishing cycles arXiv:math/9908107

[25] L Maxim, Intersection homology and Alexander modules of hypersurface complements, Comment. Math. Helv. 81 (2006) 123-155 MR2208801

[26] J Milnor, A duality theorem for Reidemeister torsion, Ann. of Math. 76 (1962) 137-147 MR0141115

[27] J Milnor, Whitehead torsion, Bull. Amer. Math. Soc. 72 (1966) 358-426 MR0196736

[28] J Milnor, Infinite cyclic coverings, from: "Conference on the topology of manifolds", Prindle, Weber \& Schmidt, Boston (1968) 115-133 MR0242163

[29] C Sabbah, Modules d'Alexander et $\mathscr{D}$-modules, Duke Math. J. 60 (1990) 729-814 MR1054533

[30] M Saito, Introduction to mixed Hodge modules, from: "Actes du colloque de théorie de Hodge", Astérisque 179-180, Soc. Math. France, Paris (1989) 145-162 MR1042805

[31] M Saito, Extension of mixed Hodge modules, Compositio Math. 74 (1990) 209-234 MR1047741

[32] J Schürmann, Topology of singular spaces and constructible sheaves, Monografie Matematyczne 63, Birkhäuser, Basel (2003) MR2031639

[33] V G Turaev, Reidemeister torsion in knot theory, Uspekhi Mat. Nauk 41 (1986) 97-147, 240 MR832411 In Russian; translated in Russian Math. Surveys 41 (1986) 119-182

[34] M Wada, Twisted Alexander polynomial for finitely presentable groups, Topology 33 (1994) 241-256 MR1273784

School of Mathematical Sciences, University of Science and Technology of China No 96, JinZhai Road, Hefei, 230026, China

Department of Mathematics, University of Wisconsin-Madison 480 Lincoln Drive, Office 713, Madison, WI 53706-1388, USA

liuyq@mail.ustc.edu.cn, maxim@math.wisc.edu http://home.ustc.edu.cn/ liuyq/, http://www.math.wisc.edu/ maxim/

Received: 13 June 2014 
